THE AstrophysicAL JouRNAL, 536:675-696, 2000 June 20

(c) 2000. The American Astronomical Society. All rights reserved. Printed in U.S.A.

\title{
THE ACTIVE JET IN NGC 4258 AND ITS ASSOCIATED SHOCKS ${ }^{1}$
}

\section{G. CECIL} SOAR Telescope Project, NOAO, and Department of Physics and Astronomy, University of North Carolina at Chapel Hill, Chapel Hill, NC 27599-3255;
cecil@physics.unc.edu

\author{
L. J. Greenhill ${ }^{2}$ AND C. G. DePree \\ Department of Physics and Astronomy, Agnes Scott College \\ N. NAGAR AND A. S. WILSON \\ Astronomy Department, University of Maryland \\ M. A. Dopita \\ Institute for Advanced Studies, The Australian National University, ACT 2611, Australia \\ I. PÉREZ-FouRNON \\ Instituto de Astrofísica de Canarias, Tenerife, Canary Islands, Spain \\ AND \\ A. L. ARgon AND J. M. Moran ${ }^{2}$ \\ Received 1999 August 2; accepted 2000 January 24
}

\begin{abstract}
We present images and spectra of the active jet and anomalous arms on subparsec through kiloparsec scales in the LINER/Seyfert galaxy NGC 4258 (M106). New VLBA and multiconfiguration VLA images show that, on $0.3-300 \mathrm{pc}$ scales, the jet in projection aligns with (1) the spin axis of the underlying accretion disk and (2) two radio hot spots $24^{\prime \prime} \mathrm{S}(840 \mathrm{pc})$ and $49^{\prime \prime} \mathrm{N}(1.7 \mathrm{kpc})$ from the nucleus. Hubble Space Telescope WFPC2 [N II] $\lambda 6583+\mathrm{H} \alpha$ images locate interactions between the jet and the interstellar medium (ISM). The most prominent is a pair of emission line arcs whose apices face away from the galaxy nucleus and envelop the leading edges of the radio hot spots. Ground-based (WHT) spectra with $2 \AA$ resolution confirm that the gaseous kinematics and excitation of both arcs have the spatio-kinematic structure expected for jet working surfaces with a shock velocity $350 \pm 100 \mathrm{~km} \mathrm{~s}^{-1}$. The north shock is oblique and may lie in a nuclear ionization cone. The south shock shows a detached, putative Mach disk. Models suggest that the S shock is a bow shock around a jet whose progress toward us through the galaxy ISM has stalled. This is notable because the inferred outflow axis is misaligned by $\sim 65^{\circ}$ (in three dimensions) with the spin axis of the accretion disk. Our emission line ratios and profiles diagnose the physical properties of the shocks, possible Mach disk, and thence the jets. The shocks lie at one end of a swath of kinematically disturbed gas that reaches back to the previously recognized spiral "anomalous arms," suggesting that they are linked dynamically by precession of the central engine; although claimed elsewhere to be bar shocks, the anomalous arms are probably a fossil record of changing jet activity in NGC 4258. Our results imply that the jet has recently moved a long way out of the plane of the galaxy. A deep Taurus Tunable Filter $\mathrm{H} \alpha$ image shows that discrete strands in the anomalous arms persist to galactocentric radii of at least $4^{\prime}(>8 \mathrm{kpc})$, indicating an ongoing ISM interaction. Subject headings: galaxies: individual (NGC 4258) - galaxies: ISM — galaxies: jets — shock waves
\end{abstract}

\section{INTRODUCTION}

Collimated jets that interact with the ambient ISM exhibit a characteristic shock pattern (e.g., Williams 1991; Leahy 1991). Ahead of the shocks one finds undisturbed ISM, followed by perhaps a photoionized precursor, a dense shell of compressed ISM behind the bow shock, the contact discontinuity between the two fluids, hot shocked jet gas, the Mach disk where the jet flow is thermalized, and jet gas. If a light jet propagates into a denser ISM, then the shocked jet material also expands around the jet to form a hot backflowing cocoon. The cocoon can cool by its lateral expansion and by mixing with the ambient ISM at the Kelvin-Helmholtz and Rayleigh-Taylor-unstable interface

\footnotetext{
${ }^{1}$ Based on observations made with the NASA Hubble Space Telescope, obtained at the Space Telescope Science Institute, which is operated by the Association of Universities for Research in Astronomy, Inc., under NASA contract NAS5-26555.

${ }^{2}$ Harvard-Smithsonian Center for Astrophysics.
}

between the two fluids (e.g., Steffen et al. 1997). If there is a strong density gradient in the ISM across the jet, then the jet may be only partially thermalized while it is redirected at an oblique shock.

Much of this theoretical structure has been confirmed by imaging spectrophotometry with high spatial resolution of ionized nebulae in Galactic Herbig-Haro objects (Morse et al. 1992, 1993). Unlike the situation in radio jets, the ratio of gas densities in these jets to ambient gas densities is high (with the jet flow clumped into planetary-mass gaseous "bullets") and there is no nonthermal radio emission. Both characteristics make the connection to powerful radio galaxies indirect.

However, over the past decade, bow shocks have been identified in the extended narrow-line regions (ENLRs) of certain active galaxies, and these are usually associated with radio hot spots. The ENLRs of M51 (Cecil 1988), NGC 1068 (Wilson \& Ulvestad 1987), Circinus (Veilleux \& Bland-Hawthorn 1996), and Mrk 573 (Ferruit et al. 1999) contain notable examples of bow shocks studied first from 
the ground then with the Hubble Space Telescope (HST). The flow usually excavates a path from the central engine, permitting anisotropic escape of photons from the vicinity of the engine. It is an open question as to which processnuclear photons or high-velocity shocks - energizes a given ENLR. The answer bears on the dynamical state of unresolved broad-line regions in AGNs.

In this paper we report the discovery of particularly clear examples of oblique and bow-shock complexes that are associated with a two-sided nuclear jet traced at radio wavelengths over several orders of magnitude in scale. The jet is related to a larger complex of spiral "anomalous arms" (van der Kruit 1972 and references therein) in the LINER/Seyfert galaxy NGC 4258 (M 106) that are prominent at many wavelengths. Its relative proximity $(7.2 \pm 0.3$ Mpc (Herrnstein et al. 1999), for which 0".1 = $3.5 \mathrm{pc}$ ) means that jet/ISM interactions can be studied in detail. These studies are enriched because NGC 4258 contains a (rare) $\mathrm{H}_{2} \mathrm{O}$ maser whose structure indicates the orientation of the accretion disk around the central engine, with high precision, in three dimensions (Miyoshi et al. 1995).

The anomalous arms span $5^{\prime}$ in optical line emission, 10' in radio continuum emission and are $5^{\prime \prime}-15^{\prime \prime}$ wide. In high spatial resolution radio images, the arms appear as jointed linear segments that turn progressively clockwise out to a radius of $\approx 2.5 \mathrm{kpc}$, beyond which the emission is oriented roughly east-west. The segments are embedded in diffuse plateaux of radio and X-ray emission. The arms appear to entrain and shock the ISM; the hottest shocked gas has a thermal X-ray spectrum (Cecil, Wilson, \& De Pree 1995b, hereafter CWD) and optical emission line ratios are consistent with gaseous excitation by shocks of several hundred $\mathrm{km} \mathrm{s}^{-1}$ (Cecil, Morse, \& Veilleux 1995a, hereafter CMV). Although usually interpreted as intertwined jet strands that flow close to the galaxy disk (Cecil, Wilson, \& Tully 1992, hereafter CWT), the arms have also been hypothesized to arise from bar shocks (Cox \& Downes 1996) or the leading edges of giant bubbles.

The arms have been imaged previously at resolutions $\geq 100 \mathrm{pc}$, and the jet-core has been imaged at $1.3 \mathrm{~cm}$ with $0.01 \mathrm{pc}$ resolution (Herrnstein et al. 1997). Our new VLBA and $\mathrm{VLA}^{3}$ radio images trace the jet on intermediate scales of $0.3-1 \mathrm{pc}$ and more than $30 \mathrm{pc}$, respectively. We also report the discovery with $H S T$ of shock-ionized nebulae that are associated with radio hot spots. Our new optical observations are more sensitive and have higher spatial resolution than previous ones. The bow and oblique shocks, and putative Mach disk, are well resolved and emit strong spectral lines. We describe these multifrequency observations in $\S 2$, present the results in $\S 3$, discuss the properties of the shocks in $\S 4$, place the active flow in the broader context of precessing jets in $\S 5$, and summarize our conclusions in $\S 6$.

\section{DATA ACQUISITION AND REDUCTION}

\subsection{Taurus Tunable Filter Images of the Anomalous Arms}

The anomalous arms do not project beyond the boundaries of the galaxy H I disk (van Albada 1980) in the radio

\footnotetext{
${ }^{3}$ The VLA and VLBA are facilities of the National Radio Astronomy Observatory. The National Radio Astronomy Observatory is a facility of the National Science Foundation operated under cooperative agreement by Associated Universities, Inc.
}

continuum, so it is interesting to determine to what maximum galactocentric radius they have interacted with the galaxy ISM by tracing their flow with ionized gas. An interaction at large radii would confirm the widely held view that the arms are close to the galaxy disk; the physical properties of the interaction would constrain the dynamical state of the arms.

To search for faint optical line emission at large radii, J. Bland-Hawthorn kindly obtained Taurus Tunable Filter (TTF, described in Bland-Hawthorn \& Jones 1998) images for us at the $4.2 \mathrm{~m}$ William Herschel Telescope (WHT). ${ }^{4}$ A 2 minute exposure through the "I0" order-separating filter was followed by a 20 minute exposure with the TTF red etalon also in the beam. Observation parameters are summarized in Table 1. The images have 1" FWHM, which is undersampled slightly by the 0"56, $24 \mu \mathrm{m}$ pixels of the Tek 2 $\left(1024^{2}\right)$ detector. The etalon was tuned to the systemic velocity of the galaxy in the $\mathrm{H} \alpha$ line, and to an effective bandpass of $1.4 \mathrm{~nm}$. Figure 9 of CWT shows that this bandwidth is large enough to span the $\mathrm{H} \alpha$ emission of the galaxy, yet small enough to avoid flux contamination by blueshifted $[\mathrm{N} \mathrm{II}] \lambda 6583$ emission in the $\mathrm{W}$ half of NGC 4258.

Unfortunately, the galaxy was observed in full moonlight and the two images were not "charge shuffled," so there are illumination gradients (particularly across the etalon-in image) which were not removed with sky or dome flat-field frames. As a first approximation, a linear gradient across the field was subtracted to substantially flatten the background of the etalon-in image. The etalon-out image was then scaled and subtracted from the etalon-in image to remove galaxy starlight. The result (Figs. $1 a$ and $1 b$ ) was flux-calibrated using the spectrophotometric standard star $\mathrm{BD}+332642$. We used the procedure outlined in BlandHawthorn (1995) to derive a system efficiency (including order separating filter) of $29 \%$ detectability of exoatmospheric flux.

\subsection{Radio Data}

\subsubsection{VLA Images of the Jet and Anomalous Arms}

To study the morphology of the anomalous arms within the inner few arcmin radii of the dusty galaxy disk, we have obtained new wide-field, continuum images with the VLA. We attain the best balance between sensitivity and angular resolution of the VLA by working near $1.46 \mathrm{GHz}$ because of the relatively steep radio spectrum of the inner part of the arms (Hummel, Krause, \& Lesch 1989). Our radio images are sensitive to structures on scales from $45 \mathrm{pc}$ to $1.5 \mathrm{kpc}$. These observations are summarized in Table 2 .

Three VLA data sets were calibrated independently using standard interferometric techniques and merged. Total onsource exposure was $14.5 \mathrm{hr}$. We made three images (FWHM 1"3, 4".3, and 12") to emphasize emission on different scales (Fig. 2); the peak intensities (rms noise) were 2.1 $(0.021), 4.6(0.032)$, and $12.6(0.027) \mathrm{mJy}^{\text {beam }}{ }^{-1}$, respectively. The signal-to-noise ratios of the initial images were limited by sidelobe response to eight bright background sources in the $30^{\prime}$ diameter primary beam of the antennas. To improve sensitivity, we reimaged and deconvolved each background source and NGC 4258 together.

\footnotetext{
${ }^{4}$ The WHT is operated on the island of La Palma by the Isaac Newton Group in the Spanish Observatorio del Roque de los Muchachos of the Instituto de Astrofisica de Canarias.
} 
TABLE 1

JOURNAL OF OPTICAL OBSERVATIONS OF NGC 4258

\begin{tabular}{|c|c|c|c|c|c|c|c|}
\hline \multicolumn{3}{|c|}{$H S T$ WPFC2 } & \multicolumn{5}{|c|}{$\begin{array}{c}\text { WHT } \\
\text { ISIS SPECTROPHOTOMETRY (1".2 WIDE SLIT) }\end{array}$} \\
\hline Filter & $\begin{array}{l}T \\
(\mathrm{~s})\end{array}$ & $\begin{array}{l}\text { Degree Roll } \\
\text { to V3 }\end{array}$ & Shock & $\begin{array}{c}\lambda \lambda \\
(\mathrm{nm})\end{array}$ & $\begin{array}{l}T \\
(\mathrm{~s})\end{array}$ & Grating $^{\mathrm{c}}$ & UT Epoch \\
\hline F658N ....... & $1400+1300$ & 135.0 & S & $360-516$ & $2700^{b}$ & $600 / 0.2 / 0.045$ & 1998 Dec 16 \\
\hline F658N ....... & $2 \times 1400+1300$ & 260.0 & $\mathrm{~S}$ & $620-700$ & $2700^{b}$ & $600 / 0.24 / 0.08$ & 1998 Dec 16 \\
\hline F658N ....... & $700+1600$ & 123.7 & $\mathrm{~S}$ & $320-550$ & 2700 & $300 / 0.2 / 0.045$ & 1998 Dec 17 \\
\hline F791W ...... & $2 \times 500$ & 135.0 & $\mathrm{~N}$ & $620-700$ & 2700 & $600 / 0.24 / 0.08$ & 1998 Dec 17 \\
\hline F547M ...... & $160+1000$ & 123.7 & & & & & \\
\hline F300W ...... & $600+2 \times 900$ & & & & & & \\
\hline \multicolumn{8}{|c|}{ TTF SPECTRAL IMAGING } \\
\hline $\begin{array}{l}T \\
(\mathrm{~s})\end{array}$ & $\begin{array}{l}\text { Band } \\
(\mathrm{nm})\end{array}$ & UT Epoch & & & & & \\
\hline $120 \ldots \ldots \ldots$ & 10 & 1999 Mar 5 & & & & & \\
\hline $1200 \ldots \ldots \ldots$ & 1.4 & 1999 Mar 5 & & & & & \\
\hline
\end{tabular}

${ }^{a}$ Extracted from the CADC data archive.

b $2700 \mathrm{~s}$ for each of 2 slit positions on sky.

c Grooves $\mathrm{mm}^{-1} / \operatorname{FWHM}(\mathrm{nm}) / \lambda$-sampling $(\mathrm{nm})$.

The radio image with highest spatial resolution (Fig. 2) was overlaid with the HST WFPC2 images described in $\S 2.3$. The radio image was rotated and scaled so that the flux centroids of the nucleus and $\mathrm{H}$ iI regions coincided to within 0 ".25. Two hot spots are visible along the axis of the almost north-south jet and were prime targets for the follow-up HST imaging and ground-based spectrophotometry that we describe in $\S 2.3$.

\subsubsection{VLBA Images of the Jet on Subarcsecond Scales}

We used the VLBA at two epochs (Table 2) to image radio continuum emission associated with the active jet. Sky frequencies bracketed those used for the VLA observations. To boost the sensitivity of the array, we added the VLA, operating as a phased $130 \mathrm{~m}$ aperture, and the NRAO $43 \mathrm{~m}$ telescope in Green Bank, West Virginia. The data were processed with standard techniques for phasereferenced VLBI. Noise levels in the images at the two epochs were 16 and $9 \mu \mathrm{Jy}$, respectively, which correspond to brightness temperatures of $1.4 \times 10^{5} \mathrm{~K}$ and $3.9 \times 10^{4} \mathrm{~K}$.
The sky frequency at the second epoch was appropriate for the serendipitous detection of $\mathrm{OH}$ maser emission but none was observed at the $2 \mathrm{mJy}(6 \sigma)$ level for $6 \mathrm{~km} \mathrm{~s}^{-1}$ spectral channels.

We combined the two VLBA images with a common 0 ."012 $\times 0.008$ beam and a signal-to-noise weighted average (see Fig. 3); we obtained a mean peak intensity of 0.23 mJy beam ${ }^{-1}( \pm 20 \%)$. The integrated intensity was $0.5 \mathrm{mJy}$, which is only $25 \%$ of the flux density observed over the inner 1".3 with the VLA. The source position is measured relative to background radio sources (see Table 2) whose positions are known to better than 0.0010 . However, a measured 0 ".0025 difference in the fitted centroid position of the northern emission component between the two epochs may be due to changing source structure in NGC 4258 or to unmodeled variations in the troposphere and ionosphere (i.e., a few parts in $10^{7}$ of the $2^{\circ}-3^{\circ}$ separation from the calibration sources. The data from the second epoch were calibrated using two phase-reference sources separately and together; the measured component position differed by only

TABLE 2

JOURNAL OF VLBI AND VLA RADIO OBSERVATIONS OF NGC 4258

\begin{tabular}{|c|c|c|c|c|c|}
\hline \multirow[b]{2}{*}{ PARAMETER } & \multicolumn{2}{|c|}{ VLBI } & \multicolumn{3}{|c|}{ VLA } \\
\hline & 1 & 2 & 1 & 2 & 3 \\
\hline Central frequency $(\mathrm{GHz}) \ldots \ldots$ & 1.413 & 1.667 & 1.44615 & 1.4649 & 1.4649 \\
\hline Bandwidth $(\mathrm{MHz}) \ldots \ldots \ldots \ldots \ldots$ & 64 & 64 & 12 & 50 & 50 \\
\hline Epoch $\ldots \ldots \ldots \ldots \ldots$ & 7 Jan 97 & $15 \mathrm{Jul} 97$ & 28 Aug 95 & $11 \operatorname{Dec} 87^{b}$ & $22 \operatorname{Mar} 88^{\mathrm{b}}$ \\
\hline Array...$\ldots \ldots \ldots \ldots \ldots \ldots \ldots$ & $\begin{array}{c}\text { VLBA } \\
+ \text { phased-VLA } \\
+ \text { NRAO140a }\end{array}$ & $\begin{array}{c}\text { VLBA } \\
+ \text { phased-VLA } \\
+ \text { NRAO140 }\end{array}$ & A & B & $\mathrm{C}$ \\
\hline Time on-source $(\mathrm{hr}) . \ldots \ldots \ldots . .$. & & & 4.6 & 3.6 & 6.3 \\
\hline Phase Calibrator (s) ........... & $1216+487$ & $\begin{array}{l}1216+487 \\
1221+464\end{array}$ & $1216+487$ & $1216+487$ & $1216+487$ \\
\hline $\begin{array}{l}\text { Beam }(\operatorname{arcsec}) \ldots \ldots \ldots \ldots \ldots \ldots \\
\text { Beam }(d e g) \ldots \ldots \ldots \ldots \ldots \ldots \ldots\end{array}$ & $\begin{aligned} 0.010 & \times 0.0070 \\
& -4\end{aligned}$ & $\begin{array}{c}0.013 \times 0.0086 \\
-11\end{array}$ & $1.3,4.3,12$ diameter & & \\
\hline
\end{tabular}

a $140 \mathrm{ft}$ telescope of the NRAO in Green Bank, WV. No useful data in January due to technical problems.

${ }^{b}$ Raw data extracted from the VLA archive and reprocessed. (Original P. I.'s were P. Crane \& S. Caganoff). 

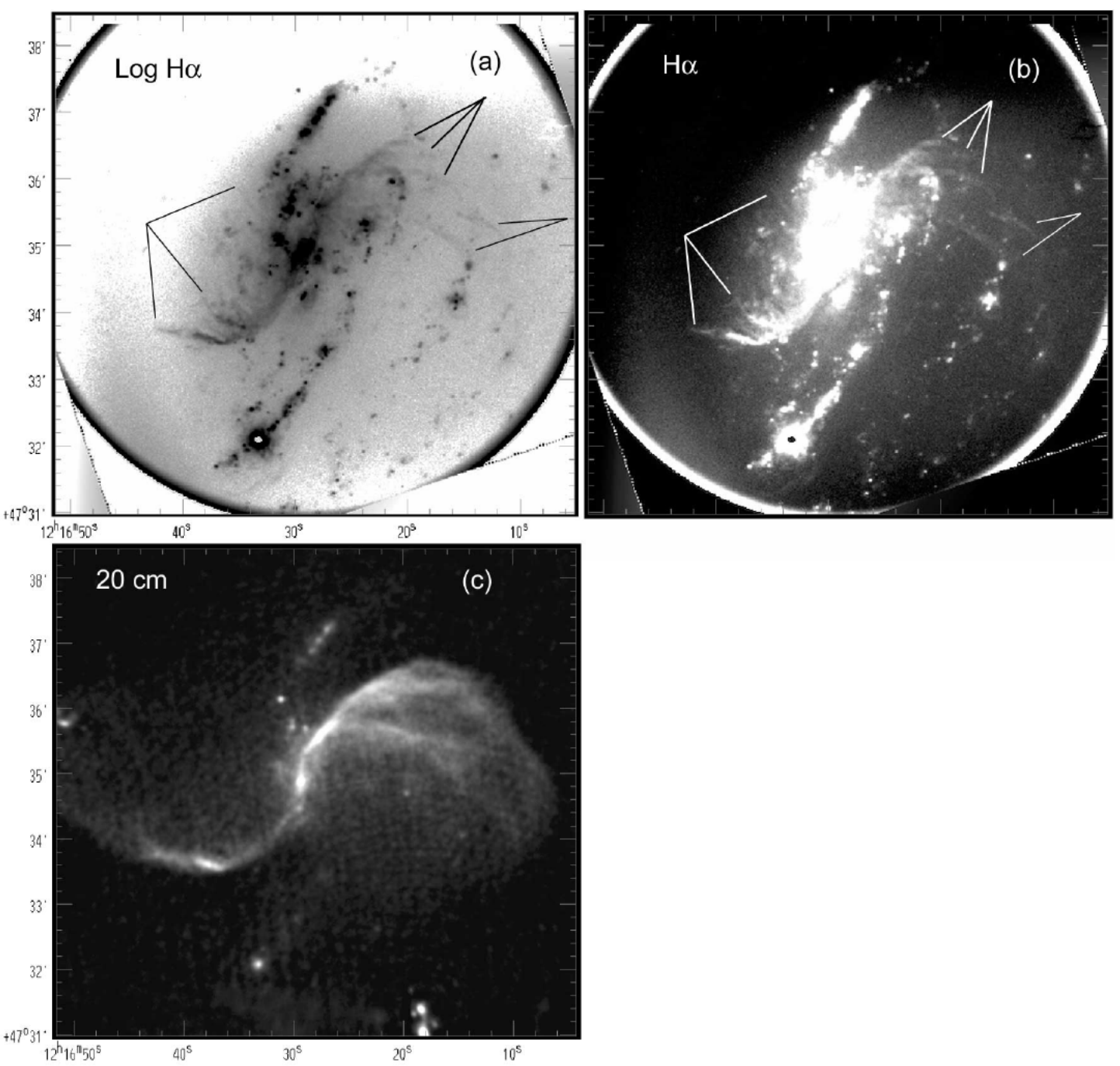

FIG. 1.-Anomalous arms of NGC 4258; $\mathrm{N}$ is at top and W at right. (a) TTF image with $1.4 \mathrm{~nm}$ bandpass centered on the galaxy systemic velocity in H $\alpha$ and 1" FWHM resolution. The intensity scale is logarithmic. An illumination gradient that was not removed by the flat fields is evident in this image. Faint linear filaments (which correspond to the well-known radio filaments) are marked in the W half, as well as extensions of known optical filaments in the E. (b) Same data as (a) but displayed with a linear, inverted gray scale to emphasize faint emission. (c) $20 \mathrm{~cm} \mathrm{~A} \mathrm{+} \mathrm{B} \mathrm{+} \mathrm{C} \mathrm{configuration} \mathrm{VLA} \mathrm{image} \mathrm{at} \mathrm{4"} \mathrm{FWHM}$ resolution, weighted to show low surface brightness features in the radio "plateaux" that trail the jet (in the sense of galaxy rotation).

0".0004, which suggests that the influence of structure within the reference sources was negligible.

\subsection{WFPC2 Images of the Anomalous Arms at High Resolution \\ 2.3.1. Observations}

We used the WFPC2 imager on HST on 1998 July 27 and November 13 to study the interaction of the multistranded jet with the galaxy ISM. Observation parameters are given in Table 1 . We have supplemented our new deep $\mathrm{H} \alpha+[\mathrm{N} \mathrm{II}]$ and continuum images with four WFPC2 GTO frames (F658N and F574M, P.I. H. Ford) and three GO frames (F300W, P.I. G. Schmidt) that we extracted from the Canadian Astronomical Data Center (CADC). At the time, the CADC was the only archive to apply "on-thefly" optimal calibrations during data extraction. Together, these images at three spacecraft roll angles and pointings span most of the optical emission of the multistranded jet. Coverage of the extremities of the NW jet is unchanged from the archive exposures, which span roughly the right half of Figure 2.

\subsubsection{Reductions}

Hot pixels were minimized by automatically reprocessing all raw images with the appropriate superdark frames; other calibrations were standard for the WFPC2 pipeline. Contamination by cosmic rays was minimized by using at least two exposures at each roll angle. One of our pointings and the pointing of all the archive exposures placed the galaxy nucleus on the PC chip. Our five F658N line and two F791W continuum frames were combined with the two F658N line and two F547M continuum archive frames by first subsampling linearly each original PC-frame pixel on a $2 \times 2$ grid, then registering the flux centroids of compact structures to a fraction of a subpixel to maximize spatial resolution around the nucleus. The WFC frames were combined in the same way. In both cases, frames were registered with common stars, thereby forming F658N line and combined F547M + F791W images. We shall refer to the latter as the continuum mosaic image. The F547M and F791W continuum frames straddle $\mathrm{H} \alpha+[\mathrm{N}$ II] in wavelength but each covers only half of the galaxy. To span the entire galaxy these frames were combined with weights set by their 


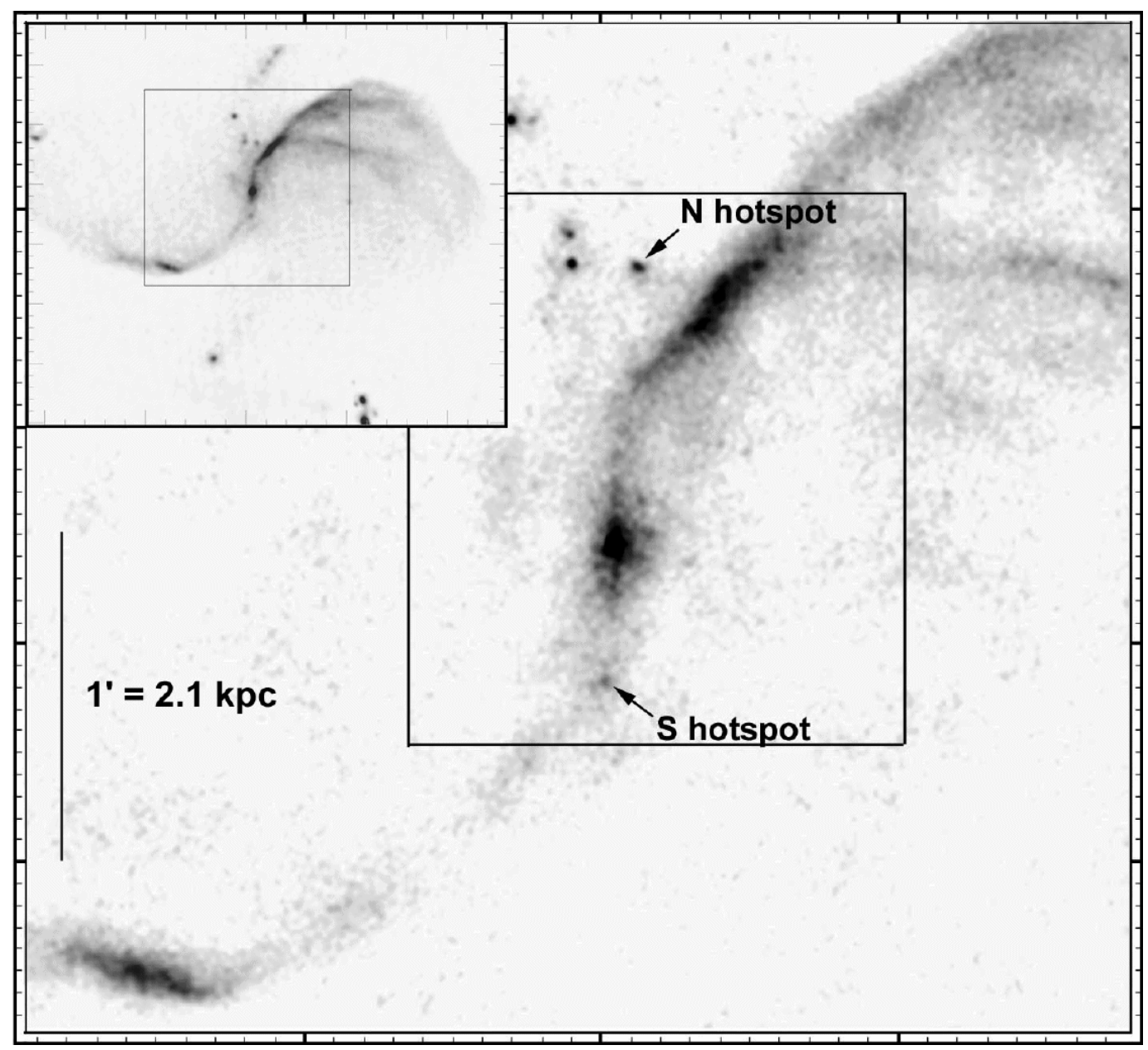

Fig. 2. $-20 \mathrm{~cm} \mathrm{~A} \mathrm{+} \mathrm{B} \mathrm{+} \mathrm{C} \mathrm{configuration} \mathrm{VLA} \mathrm{image} \mathrm{of} \mathrm{NGC} 4258$ with 1".3 resolution FWHM. The field of view is 3'.1 $\times$ 3'.1. Ticks are shown every 4", with $\mathrm{N}$ at top. Note the almost north-south, two-sided jet near the nucleus and how it is directed at two putative hot spots. The region plotted in Fig. 10 is delineated. The main image at full resolution (1"3) emphasizes emission near the nucleus. The inset image, also shown as Fig. 1c, shows a larger field of view $\left(7^{\prime} \times 6.5\right)$ at lower $\left(4^{\prime \prime} 3 \mathrm{FWHM}\right)$ resolution and clarifies the radio emission at low surface brightness.

relative filter efficiencies for an object with Sbc galaxy colors. This choice was correct as judged by smooth isophotes at all flux levels in the combined continuum image. The results were normalized by their exposure times to produce data-number-counts $\mathrm{s}^{-1}$ images.

The combined continuum mosaic image was scaled by the relative transmission efficiencies of the F547M + F791W and F658N filters for an Sbc galaxy (see Table 6.2 in Biretta 1996) and subtracted from the line image. This produced a continuum-free $[\mathrm{N} \mathrm{II}]+\mathrm{H} \alpha$ image that was then converted to an absolute flux scale using an electronic gain of $7 e^{-} /$ data-number and the efficiency plots in Table 6.1 of the Handbook. We did not correct for parallel charge transfer inefficiencies in the WFPC2 CCDs. Line and continuum images are shown together in color in Figure 4, while Figure 5 shows the inner part of the $\mathrm{H} \alpha+[\mathrm{N} \mathrm{II}] \mathrm{PC}$ image, and its inserts show ionized nebulae on the leading edges of the radio hot spots.

\subsubsection{Corrections for Filter Transmission}

Gaseous velocities in this galaxy have been fully mapped by imaging Fabry-Perot (FP) spectrophotometry (CWT); these data also mapped the [N II] $\lambda 6583 / \mathrm{H} \alpha$ flux ratios SE of the nucleus. Based on this data set, we constructed an image representing the relative transmissions of $[\mathrm{N} \mathrm{II}]$ $\lambda 6583$ and $\mathrm{H} \alpha$ through the $\mathrm{F} 658 \mathrm{~N}$ filter wherever there is significant line emission. Accounting for the sometimes broad lines (few hundred $\mathrm{km} \mathrm{s}^{-1}$ line FWZI) outside the nucleus we find that the $[\mathrm{N} \mathrm{II}] \lambda 6583$ line is usually close to the peak of the $\mathrm{F} 658 \mathrm{~N}$ filter transmission while $\mathrm{H} \alpha$ is on the blue wing of that filter (see $\S 3.5$ ). The FP spectra show that line emission associated with the anomalous arms has [N II] $\lambda 6583 / \mathrm{H} \alpha$ flux ratios between 0.5 and 0.8 . So, everywhere except in the NW quadrant, counts through the F658N filter come about equally from the two lines. In the NW quadrant, $\mathrm{H} \alpha$ emission is redshifted by more than 250 $\mathrm{km} \mathrm{s}^{-1}$ relative to the galaxy systemic velocity, so also approaches the peak transmission of the filter. In this way we constructed $\mathrm{H} \alpha$ fluxes at each point in the HST image. Flux calibration was confirmed by our WHT long-slit spectra of several features (see $\S 3.5$ ).

$$
\text { 2.3.2.2. Color Map }
$$

We ratioed the archival F300W image with our continuum mosaic image to produce a color map (Fig. 6b). The map shows clearly the continuum extension noted in ground-based images by Dutil, Beauchamp, \& Roy (1995) along P.A. $150^{\circ}$, high reddening in the central $4^{\prime \prime}$ diameter that must influence the near nuclear gas morphology (Fig. 5 ), and regions of star formation in the galaxy disk. 


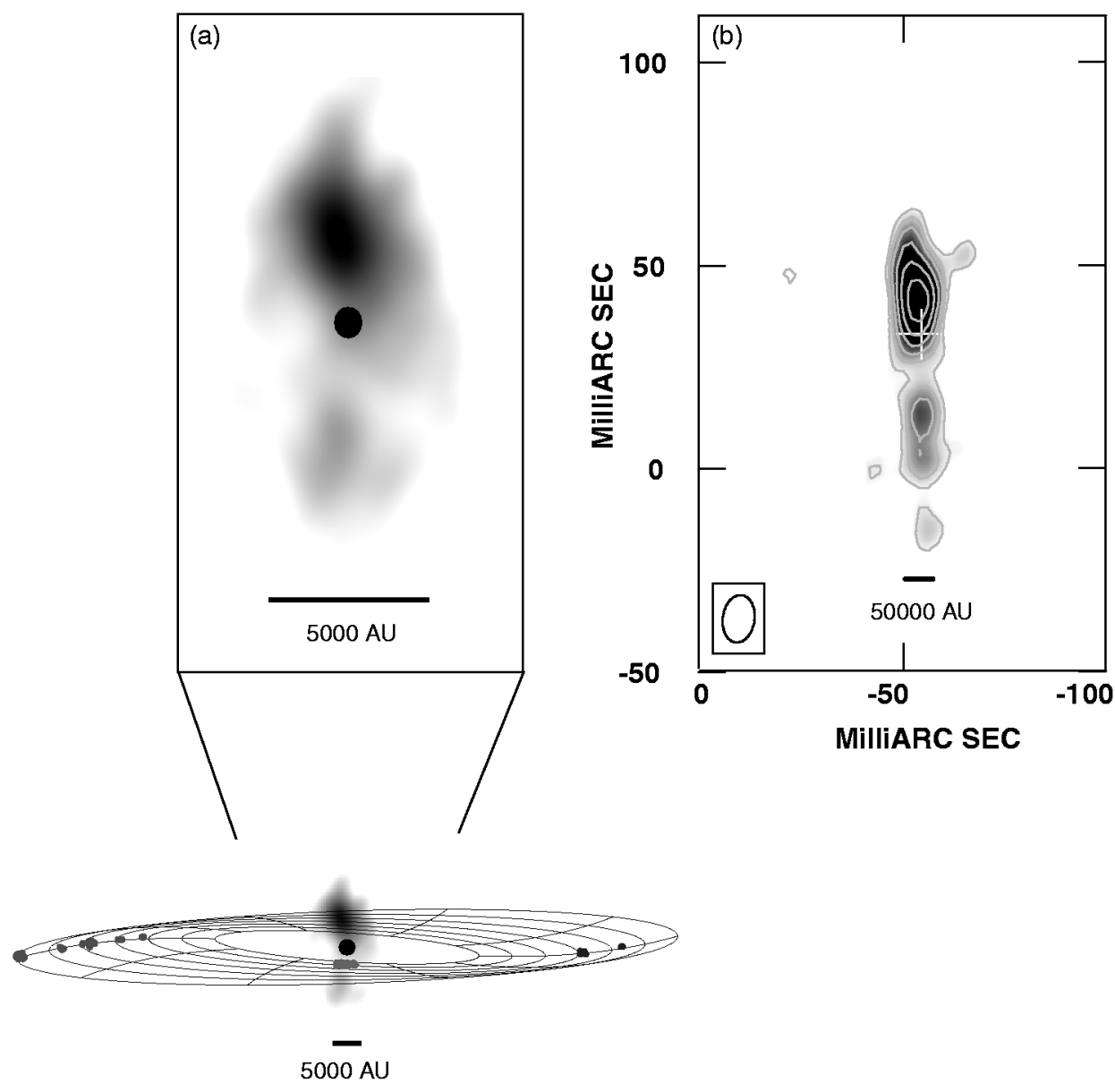

FIG. 3.-VLBA images of NGC 4258. (a) $1.3 \mathrm{~cm}$ continuum emission (gray scale) lies along the rotation axis of the accretion disk and within $\sim 4000$ Schwarzschild radii of the central engine. Peak emission is $\approx 2 \mathrm{mJy}$, although this is time variable; the lowest level emission is $0.6 \mathrm{mJy}$. Water masers trace the disk, a model of which is shown with the observed positions of the masers superposed (Herrnstein et al. 1996, 1997). (b) Combined $1.4 \mathrm{GHz}$ and $1.6 \mathrm{GHz}$ VLBA image of NGC 4258. The emission traces a more extended portion of the jet observed at $1.3 \mathrm{~cm}$. The two maps were convolved with a $0.012 \times 0.008$ beam (FWHM), shown at lower left. The lowest contour is $4 \sigma(1 \sigma=8 \mu \mathrm{Jy})$, and the levels increase by flux multiples of $\sqrt{2}$. Maser positions on the near side of the disk are marked by the cross; the uncertainty in their astrometric position is $\approx 0$ ".002. The uncertainty in the astrometric position of the low-frequency continuum is $\approx 0$ " 003 . The bar is $00^{\prime \prime} 005$ long, about the inner radius of the disk shown in $(a)$.

\subsection{Long-Slit Optical Spectrophotometry to Characterize the Radio Hot Spots}

Our HST images (e.g., Fig. 5) revealed that ionized nebulae are associated with the two radio hot spots at the ends of the active jet. We therefore conducted follow-up optical spectrophotometry from the ground to characterize the physical properties of these nebulae.

\subsubsection{Observations}

We obtained long-slit spectra of the hot spot nebulae just before the onset of astronomical twilight on 1998 December 16 and 17. The ISIS double-beam spectrometer was used at the Cassegrain focus of the William-Herschel $4.2 \mathrm{~m}$ Telescope (WHT). ISIS was configured with a dichroic beam splitter at $570 \mathrm{~nm}$ to simultaneously feed blue and red gratings used with their blazes toward the collimator and near their blaze wavelengths. See Table 1 for instrument parameters.

An EEV-42 $2 \times 4 \mathrm{~K}$ CCD (EEV12) with $13.5 \mu \mathrm{m}\left(0^{\prime \prime 2}\right)$ pixels was used in the blue, while a $1 \mathrm{~K}^{2}$ Tektronix chip (number TEK2) with $24 \mu \mathrm{m}(0,4)$ pixels was used in the red. Both CCDs are antireflection coated, have DQE greater than $70 \%$ at the wavelengths we used and were read using the standard controller setting, giving blue and red noise values of 3.9 and $5.0 e^{-}$rms, respectively. The readout regions included bias overscan as well as areas nominally unilluminated by the slit (the latter to check for scattered light, which was found to have amplitude of less than 1 data-number and so was ignored).

Using the STSDAS "metric" task, the positions of both hot spot nebulae were measured from the HST frames relative to a star also in the $H S T$ continuum frames. Using the off-slit TV guider, we centered the star on the spectrometer slit at the observation P.A., then offset blindly to the nebula. ${ }^{5}$ This was repeated for a second position (displaced $1^{\prime \prime 2} \mathrm{~N}$ of the first) at the same slit P.A. on the $\mathrm{S}$ nebula. There was time for only a single slit position on the $\mathrm{N}$ hot spot nebula; luckily this feature appears straighter in the WFPC2 image than the S nebula.

High-altitude sky conditions were not photometric (patchy cirrus and wind-blown dust) and seeing was 1".3 \pm 0 ". 1 FWHM at the typical $z=1.3$ air mass of these observations. Spectra of the $(\mathrm{N}, \mathrm{S})$ bow shocks were taken

${ }^{5}$ The star is 40.8 and $24^{\prime \prime} \mathrm{W}$ and $\mathrm{N}$ of the nucleus, respectively. The $\mathrm{S}$ nebula is $43^{\prime \prime}$ and $50^{\prime \prime} 6 \mathrm{E}$ and $\mathrm{S}$ of the star, respectively. The N nebula is $35^{\prime \prime}$ and $25^{\prime \prime} \mathrm{E}$ and $\mathrm{N}$ of the star, respectively. 


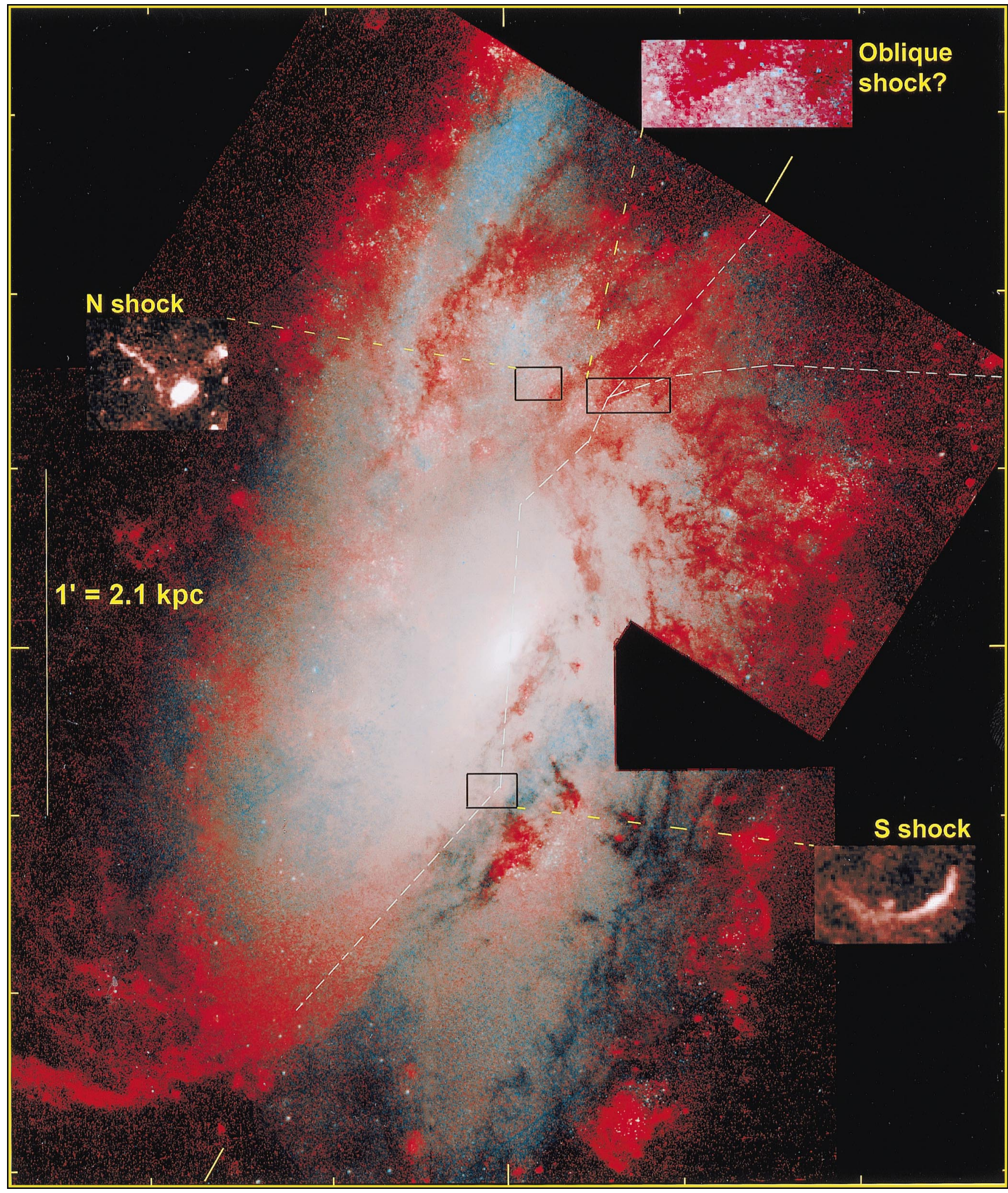

FIG. 4. - Combined $\mathrm{H} \alpha+[\mathrm{N}$ II $]$ line (red) and F547M + F791W continuum (blue/green) HST WFPC2 Images. The image spans a region 2'.9 $\times$ 3'.5 with $\mathrm{N}$ at top with ticks every $30^{\prime \prime}$. To maximize dynamic range, the square root of counts is mapped to a linear intensity scale. The kinematic line of nodes along P.A. $150^{\circ}$ is marked; the near side of the galaxy is at lower right. The anomalous arms sweep from lower left to upper right; the green lines trace the peak brightness of several strands of the radio jet. Three major regions of jet/ISM interactions are labeled. The "Oblique shock?" is tentative, pending spectroscopic confirmation of anomalous kinematics. However, this region is a prominent radio (Fig. 2) and X-ray hot spot.

along P.A. $=\left(59^{\circ}, 100^{\circ}\right)$, which straddled the parallactic angle. We calculate a maximum shift of 0 ." 3 between the blue $(\mathrm{H} \beta)$ and red $([\mathrm{S} \mathrm{II}])$ spectra, which is a small fraction of the slit width (1".2) and seeing diameter. Spectrophotometric standard stars were observed in twilight with narrow and wide slits; standard La Palma extinction curves were used and give line fluxes in good agreement with the HST images, despite nonphotometric conditions. 


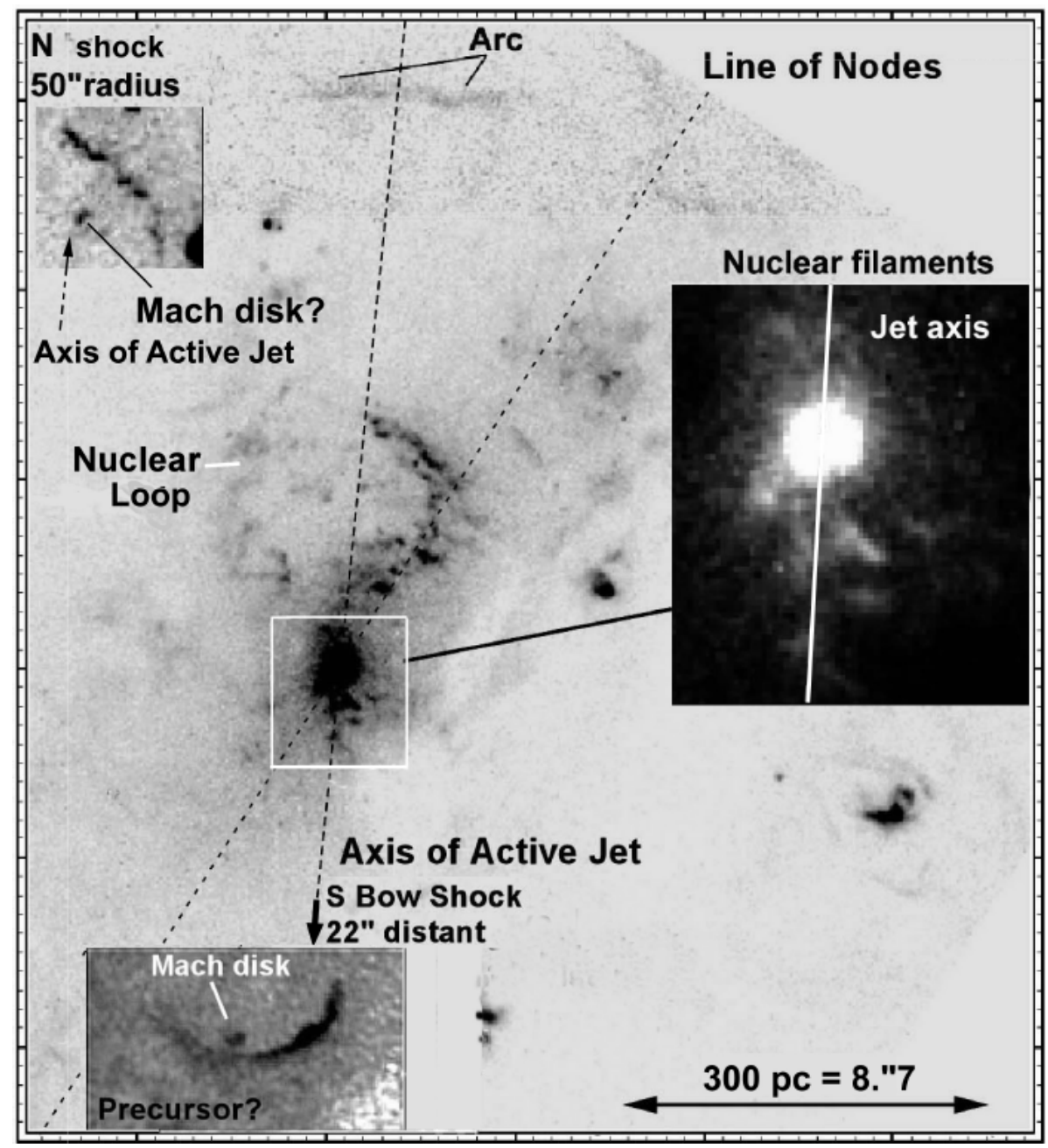

FIG. 5. - HST image of $\mathrm{H} \alpha+[\mathrm{N}$ II] emission in the nuclear region of NGC 4258. Main image: five continuum-subtracted PC chip frames were registered with subpixel interpolation for a total line exposure of $6400 \mathrm{~s}$. Ticks are shown every 0"455 (10 PC pixels) with $\mathrm{N}$ at top. Inserts: the $\mathrm{N}$ and $\mathrm{S}$ hot spot nebulae, as imaged by subpixel interpolation on WFC chips, are inserted at the same scale but closer to the nucleus than they really are. To fit in the main image, the image of the $\mathrm{N}$ shock complex has been inserted to one side of the inferred axis of the active jet; the dashed arrow continues the axis in the main image into this insert at upper left. The insert at right shows the nucleus at larger scale and with a different intensity scaling to emphasize filamentary structure. The white line marks the axis of the active jet.

\subsubsection{Reductions}

IRAF 6 "specred" reductions were used. We subtracted the average of the outer 50 positions in the long-slit images to remove sky lines. For all spectra we used the IRAF "background" task to model the starlight continuum away from spectral lines with third-order Chebyshev polynomials for each position along the slit. This was adequate for the red spectra because of the large equivalent widths of most red emission lines at the locations of the hot spot nebulae. Line profiles in the blue spectra were more strongly influenced by underlying stellar absorption. To reduce this, we averaged the $\mathrm{S}$ spectra at positions $51-100$ along the slit to form a mostly absorption spectrum. Narrow, residual $\mathrm{H} \beta$ emission was excised, then the result was scaled in intensity to reduce absorption around the $\mathrm{H} \beta$ and [O III] $\lambda \lambda 4959$, 5007 complex. Radial velocities were corrected to heliocentric values using the IRAF "rvcorrect" task.

${ }^{6}$ IRAF is distributed by the National Optical Astronomy Observatories, which are operated by AURA, Inc., under contract to the National Science Foundation.
The resulting sky and starlight-subtracted spectra are shown in Figures 7 and 8 for $\mathrm{S}$ and $\mathrm{N}$ nebulae, respectively. The WHT has a reputation for precise offsets within its Cassegrain guider field. This is illustrated in our spectra of the $\mathrm{S}$ nebula by the absence in the first exposure of a 0.5 diameter knot (Fig. 7a) visible in the WFPC2 image ( $(4.2)$, and its appearance in the second exposure (Fig. 7b). Figure 9 shows spectra that were extracted along rows of Figure 7. The large kinematic excursions at abrupt boundaries indicate that strong shocks are present in both nebulae.

\section{EMPIRICAL RESULTS}

In this section we examine correlations between radio and optical line emission on several scales. We discuss the physical properties of the ionized nebulae associated with the anomalous arms and the radio hot spots.

\subsection{Faint Jet Strands}

The deep H $\alpha$ TTF image shows discrete ionized filaments that coincide with strands of radio emission (Fig. 1). Evidently, ionized gas is energized by the flow out to nearly $4^{\prime}$ 


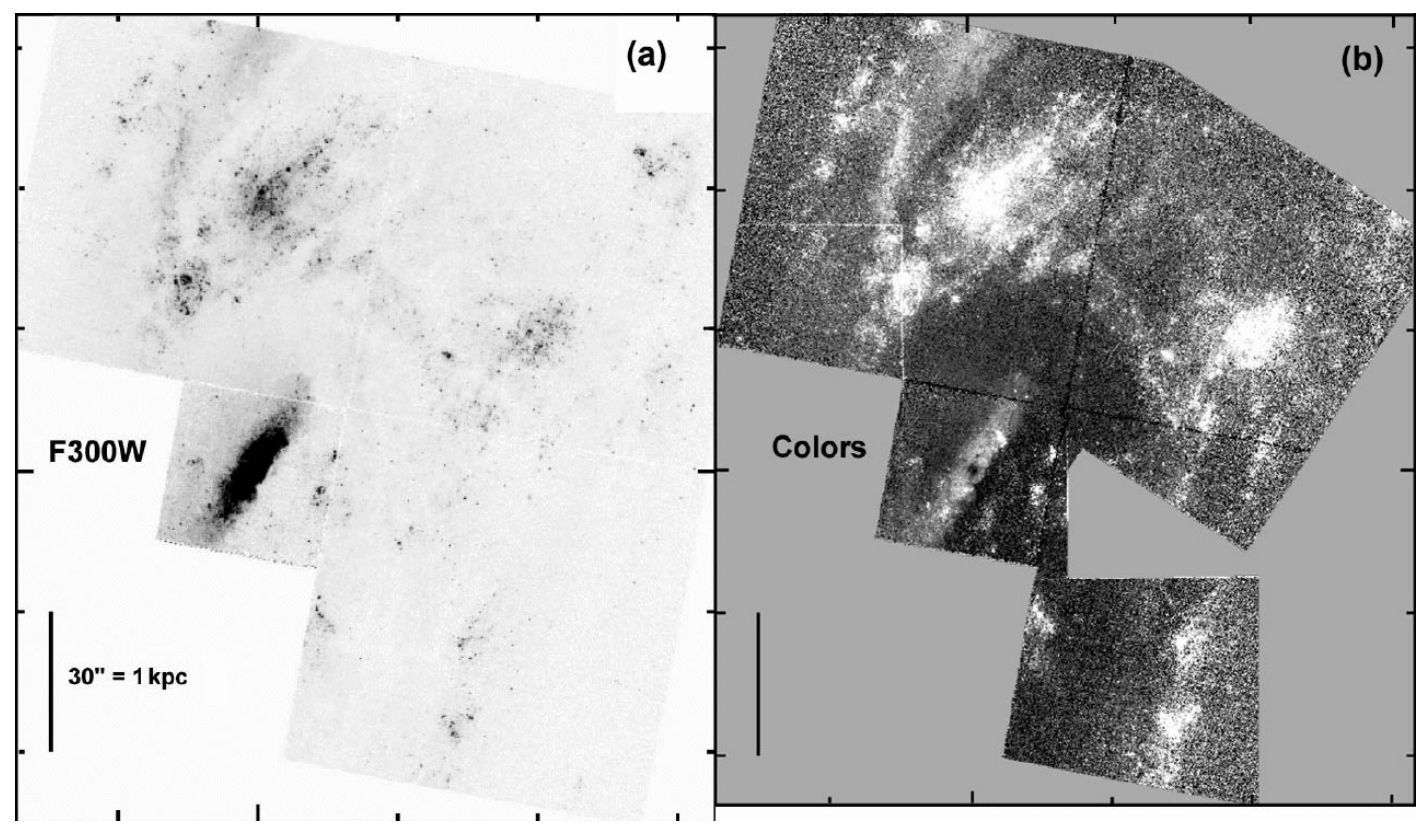

FIG. 6.- (a) Archival HST F300W WFPC2 image. Ticks are shown every 4", with N at top. (b) Color map formed from the ratio of this image and our F547M + F791W continuum mosaic. Lighter is bluer, darker is redder. The extension along P.A. $150^{\circ}$ (close to the line of nodes) is the continuum discussed by Dutil et al. (1995). Only the NW anomalous arm is faintly visible in $(b)$.

radii on the sky. The present data do not constrain kinematics, but we find that the E filaments have $\mathrm{H} \alpha$ surface brightness $\quad(0.6-2.5) \times 10^{-16} \quad \operatorname{ergs~s}^{-1} \mathrm{~cm}^{-2} \operatorname{arcsec}^{-2}$. Assuming case $\mathrm{B}$ recombination conditions at $10^{4} \mathrm{~K}, n_{p} \approx$ $0.9 n_{e}$, and no reddening, we found an electron density $n_{e}=$ $1.8 \times 10^{12}\left(L_{\mathrm{H} \alpha} / V f\right)^{1 / 2} \mathrm{~cm}^{-3}$ for gaseous filling factor $f$, emitting volume $V$, and $\mathrm{H} \alpha$ luminosity $L_{\mathrm{H} \alpha}$. Assuming a cylindrical emitting volume and $L_{\mathrm{H} \alpha}=(3.4-13) \times 10^{35} \mathrm{ergs}$ $\mathrm{s}^{-1} \operatorname{arcsec}^{-2}$, we found $n_{e} \approx 1.5 \pm 0.5 f^{-1 / 2} \mathrm{~cm}^{-3}$ in the faint filaments. This is roughly half the average value along the strands of the SE jet (CWT), but consistent with values expected in the galaxy disk. The recombination time is so short - (7-17) $\times 10^{4} \sqrt{f} \mathrm{yr}$ - that the interaction must still be ongoing. The total mass of ionized gas in a typical filament, considering the contributions of $\mathrm{H}$ and $\mathrm{He}$ only, is $M_{\mathrm{ion}}=\left(n_{p} m_{p}+n_{\mathrm{He}} m_{\mathrm{He}}\right) V f$, which (with the approximation $n_{\mathrm{He}} \approx 0.1 n_{p}$ and $\left.n_{e} \approx n_{p}+1.5 n_{\mathrm{He}}\right)$ reduces to $M_{\mathrm{ion}}=$ $1.2 n_{e} m_{p} V f=10^{-57} n_{e} V f^{p} M_{\odot}$. A typical filament $4^{\prime \prime}$ wide and $\approx 40^{\prime \prime}$ long, when approximated as a cylinder, has $M_{\text {ion }} \approx 10^{5} \sqrt{f} M_{\odot}$. We discuss the implications in $\S 5$.

\subsection{The North-South Inner Jet}

We processed our highest angular resolution VLA image (Fig. 2) to emphasize compact emission near the central engine. Between $\approx 1^{\prime \prime}$ and $20^{\prime \prime}$ from the central engine, the width of the jet is smaller than the 1".3 resolution. The inner jet lies along P.A. $=-3^{\circ} \pm 1^{\circ}$, which is consistent with the projected spin axis of the accretion disk traced by the $\mathrm{H}_{2} \mathrm{O}$ masers (P.A. $=-4^{\circ} \pm 2^{\circ}$; Miyoshi et al. 1995). The compact $1.3 \mathrm{~cm}$ jet-core emission observed within $0.02 \mathrm{pc}$ of the dynamical center of the "maser accretion disk" also lies along P.A. $\sim 0^{\circ}$, but a precise estimate is difficult due to uncertainty in the location of the center (of order $0.003 \mathrm{pc}$ east-west) and partial obscuration of the southern $1.3 \mathrm{~cm}$ emission by the disk.

The north-south radio continuum structure is almost certainly nonthermal synchrotron emission that extends con- tinuously and almost linearly from the central engine. This observation stands in contrast to the Cox \& Downes (1996) model of inflow along bar shocks or the Plante et al. (1991) model of a channeled jet.

The SW quadrant of the galactic disk is nearest us based on the pattern of dust obscuration and on analyses of $\mathrm{H} \mathrm{I}$ data (van Albada 1980). We may infer that the northern jet lies above the galactic disk because (1) the maser accretion disk is tipped down $\sim 7^{\circ}$ at its innermost radius (Miyoshi et al. 1995), and (2) at $1.3,18$, and $21 \mathrm{~cm}$, the northern continuum emission is stronger. Herrnstein et al. (1996) show that ionized material around the disk can partially obscure the southern jet emission at small impact parameters. Presumably, the disk extends to larger separations, and there it could also obscure the long-wavelength emission. The material must lie up to $\approx 00^{\prime \prime} 05$ (projected) from the central engine $(\approx 2 \mathrm{pc}$ deprojected $)$ for an inclination of $82^{\circ}$. Because of the long-wavelength, free-free absorption demands only a modest emission measure of $8 \times 10^{6}$ $\mathrm{pc} \mathrm{cm}{ }^{-6}$, which corresponds to a mean electron density of $\approx 2 \times 10^{3} \mathrm{~cm}^{-3}$ over $2 \mathrm{pc}$ at a temperature of $10^{4} \mathrm{~K}$. We note that $2 \mathrm{pc}$ is also a reasonable approximate upper limit for the extent of obscuring material, assuming it has dust, because $2 \mu \mathrm{m}$ imaging (Chary et al. 2000) and PSF fits to archival F502N continuum WFPC2 images (Barth et al. 1999) show only a pointlike nucleus on 0 ". 1 scales.

\subsection{Correlations of Optical and Radio Emission 3.3.1. Overview}

The VLA image with highest resolution (Fig. 2) shows that the radio emission $\mathrm{S}$ of the nucleus breaks into three distinct regions: the north-south outflow from the central engine terminates at an obvious hot spot $24.5 \mathrm{~S}$ of the central engine, a gently curved segment then takes off from the $\mathrm{S}$ hot spot at an $\approx 25^{\circ}$ projected angle for another $80^{\prime \prime}-$ $90^{\prime \prime}$, and finally an extended ridge of radio emission appears as the arm turns through east-west on the sky. The HST 

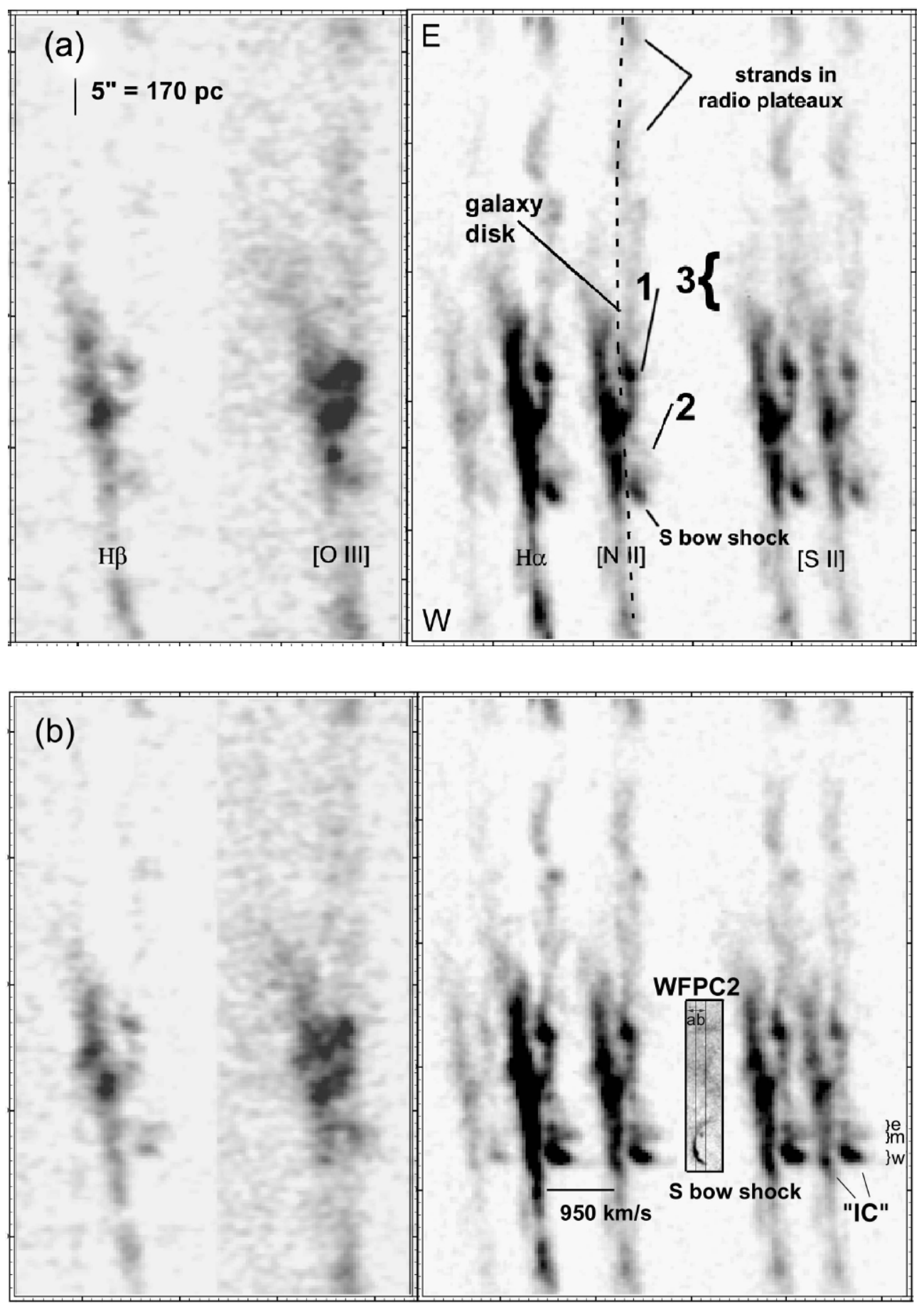

FIG. 7.- Montage of emission line profiles across the $\mathrm{S}$ shock complex including (in the bottom third of each panel) the $\mathrm{S}$ hot spot nebula, for different ions at two adjacent sky positions $(a)$ and $(b)$ after subtracting sky lines and galaxy starlight. Position $(b)$ is 1 ."2 N of position $(a)$, as shown in the HST WFPC2 image inserted in the right-hand panel of $(b)$. This image has the same scale as the slit spectra and shows the putative Mach disk to be discussed in $\S 4.2$ within the S nebula. Spectra were obtained along $100^{\circ}$ P.A.; the total vertical extent is 1'.5. Left panels: blue spectra ([O II $]$ omitted). Right panels: red spectra ([O I $]$ omitted). Note differences in the excitation distribution of [O III] $\lambda 5007$ compared to the other lines. The dashed curve in the right panel of $(a)$ shows the inferred bar-forced rotation curve for the $[\mathrm{N} \mathrm{II}] \lambda 6583$ line (CWT). The three spectra in Fig. 9 were extracted from the regions shown in the right panel of $(b)$. The "IC" pattern referred to in the text is marked. Regions 1-3 are discussed in $\S 3.6$.

WFPC2 image (Fig. 10) shows filamentary line emission associated with the last two structures, but nothing associated with the active jet until the $\mathrm{S}$ hot spot.

The locus in radio emission of the NW arm is roughly reflection symmetric with the SE arm. However, the flux distributions of the two arms are quite different. The hot spot $49^{\prime \prime} \mathrm{N}$ of the nucleus is displaced $3^{\prime \prime} \mathrm{W}$ (trailing direc- tion of the galaxy rotation) from the jet axis defined by the $S$ hot spot and nucleus. Meanwhile, the NW anomalous arm has an extended ridge (at the point where the SE arm is very faint), then appears to bifurcate.

The HST figure confirms previous ground-based images that line-emitting filaments are more extensive in the SE quadrant than in the NW. The strands have comparable 


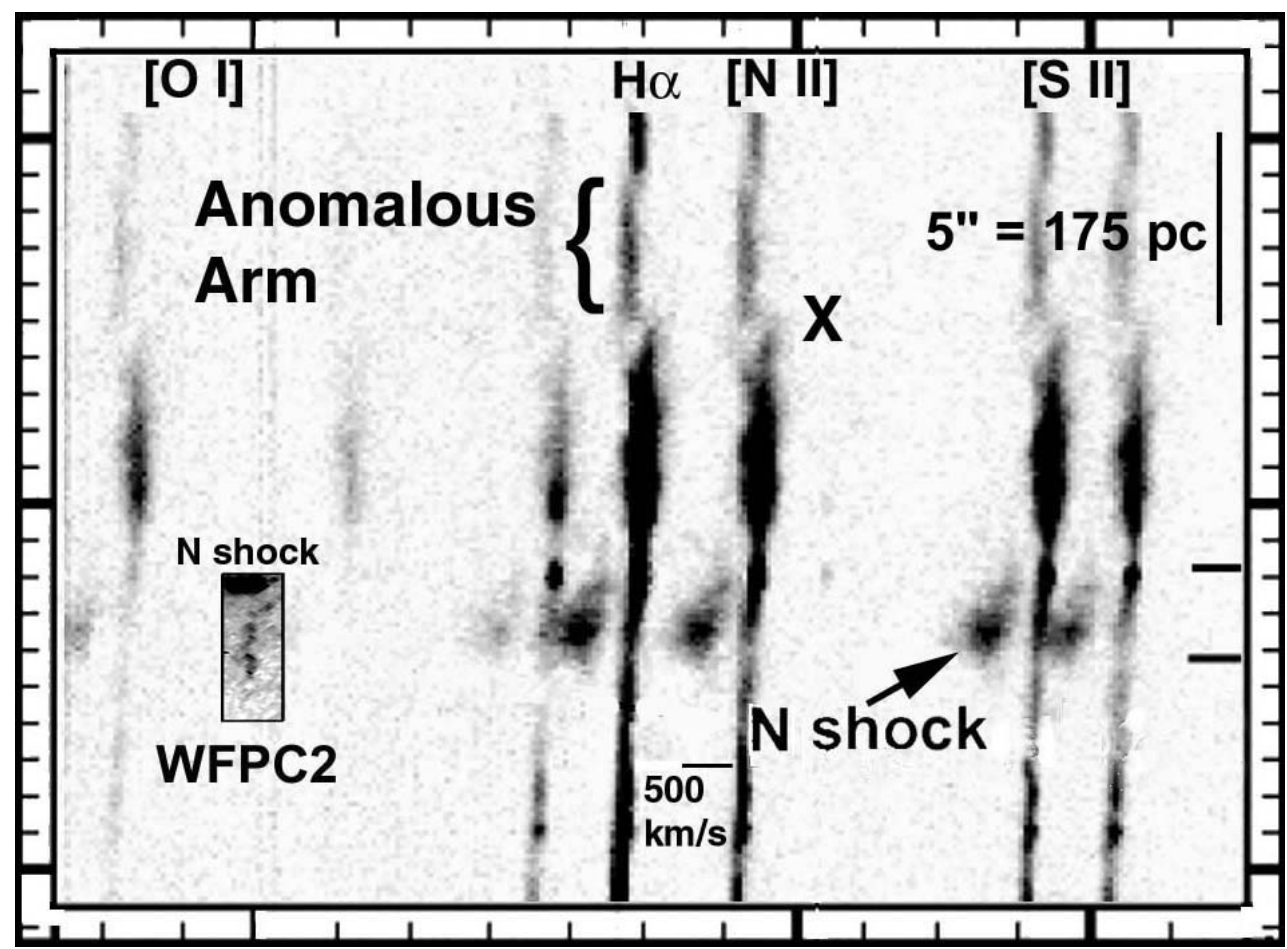

FIG. 8. - Red spectrum, including the $\mathrm{N}$ hot spot nebula, after subtracting sky lines and galaxy starlight. The slit lay along P.A. $=59^{\circ}$ with SW at top. The plot spans $25^{\prime \prime}$ vertically. The WFPC 2 image is inserted at the same scale for reference. The line peak of the $\mathrm{N}$ nebula is blueshifted $\approx 360 \mathrm{~km} \mathrm{~s}^{-1}$ relative to the background local disk motion. Note the kinematic discontinuity in region "X," as the slit begins to cut across the $\mathrm{N}$ anomalous arm. The spectrum plotted in Fig. 11 was summed between the horizontal lines on the right-hand side of this plot.

width in the radio and HST images and are clearly resolved by $H S T$ into twisted, knotted structures that resemble vortices seen in terrestrial smoke plumes. Ground-based FP spectra (CWT) show that the strands that form the SE anomalous arm oscillate spatially and kinematically. The strands separate cleanly when the oscillation amplitudes drop to $\pm 100 \mathrm{~km} \mathrm{~s}^{-1}$ and increase to $\pm 5^{\prime \prime}$, at radii of more than $25^{\prime \prime}$. Our HST image does not show helical strands because its filter bandpass is much larger than that of the FP, see Figure 19 of CWT. Helices are also absent in the
VLA images, despite sufficient resolution and flux sensitivity.

\subsubsection{Radio Hot Spots}

Figure 10 is an overlay of the radio and optical data sets and shows emission line nebulae associated with both radio hot spots. The $\mathrm{S}$ hot spot is circular and is associated with an enveloping arc in the optical line-emission image. The $\mathrm{N}$ radio hot spot is flattened on its leading edge, the side with line emission. The flux densities of both hot spots and the

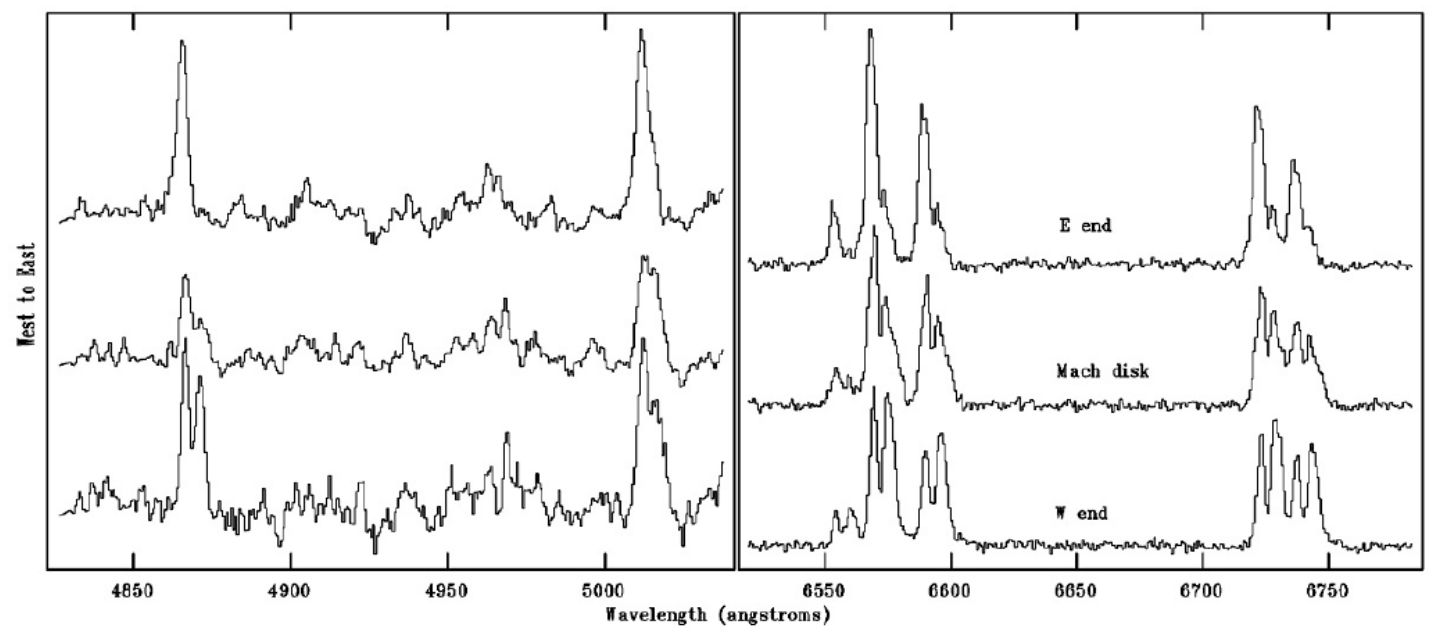

FIG. 9. - Spectra of the S nebula averaged between the braces shown at the right-hand side of Fig. $7 b$. Red spectra are unsmoothed while blue have been smoothed with a 3 pixel boxcar. Each emission line has two velocity systems separated by $275-300 \mathrm{~km} \mathrm{~s}^{-1}$. The red system has a velocity dispersion of $215-280 \mathrm{~km} \mathrm{~s}^{-1} \mathrm{FWHM}$, while the blue has $125 \mathrm{~km} \mathrm{~s}^{-1}$. 


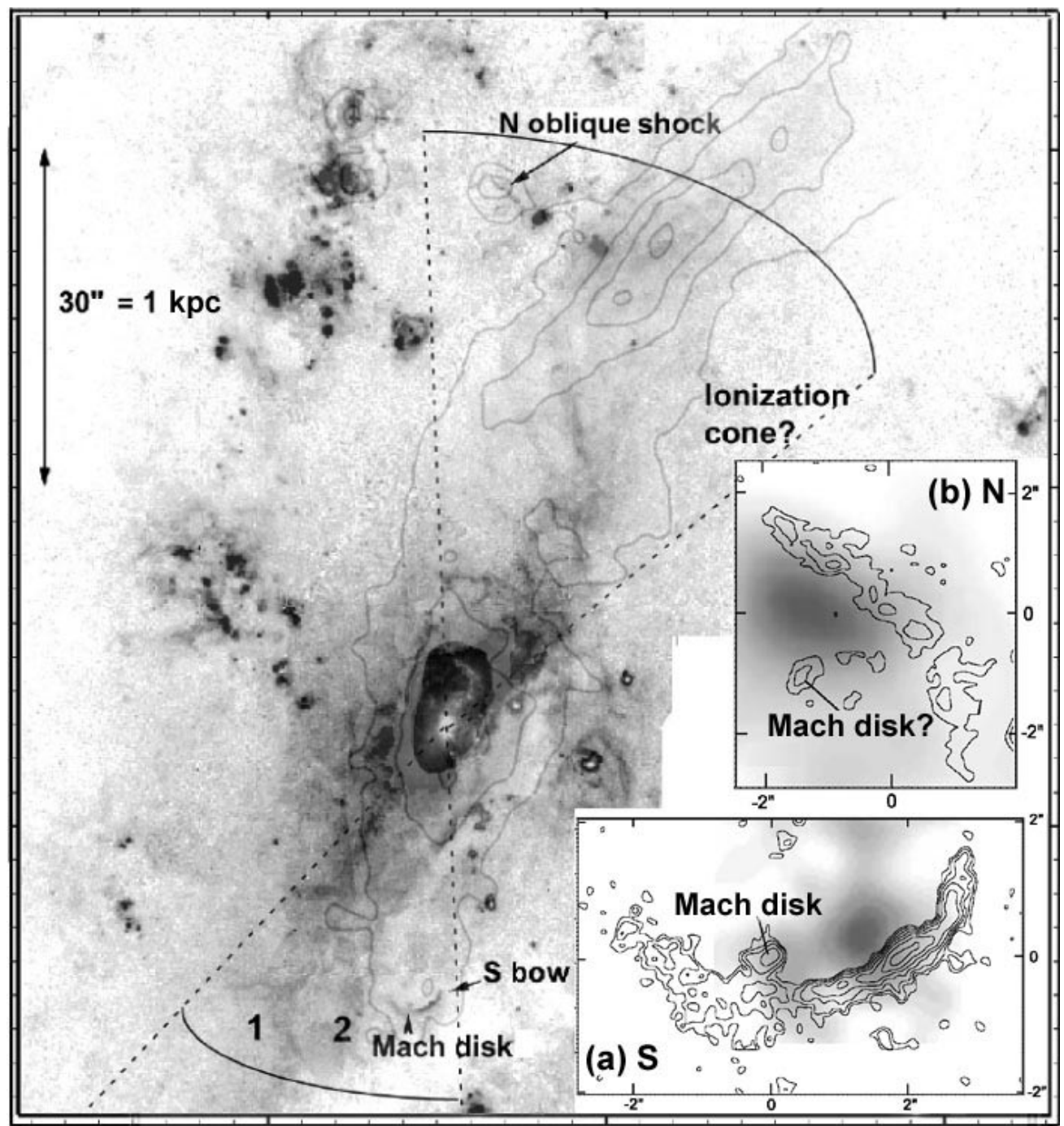

FIG. 10.-Comparison of our $H S T \mathrm{H} \alpha+[\mathrm{N}$ II] image and VLA radio continuum image. Due to the large dynamic range of the data, intensities in the HST image are log-scaled and the nucleus is shown with a different transfer function and gray scale (cf. Fig. 5). The VLA image at 4".3 FWHM resolution, processed to emphasize the linear jet, is as contours with constant flux spacing. N is at top with ticks every $3^{\prime \prime}$. Spectra of regions 1 and 2 are shown in Fig. $7 a$. Region 1 is a faint arc with high velocities; region 2 shows broad emission lines between the Mach disk and region 1. Dashed lines delineate features within the intersection of the putative ionization cones with the large-scale galaxy disk, see $\S 4.3$. The $\mathrm{S}$ cone is speculative. Inserts at right: the $\mathrm{S}$ and $\mathrm{N}$ shock complexes in [N II] $26583+\mathrm{H} \alpha$ line emission, imaged by combining five subpixel interpolated WFC images. The contours start at a surface brightness of $3 \times 10^{-15} \mathrm{ergs} \mathrm{s}^{-1} \mathrm{~cm}^{-2}$ arcsec ${ }^{-2}$, then increase by a multiplicative factor of $\sqrt{2}$. The narrow dimensions are unresolved. The $20 \mathrm{~cm}$ radio hot spots are shown with 1".4 FWHM resolution in inverted gray scale. The radio and line data sets are registered to an estimated $2 \sigma$ uncertainty of 0 ".2. The Mach disk in $(a)$ is established with high confidence. The candidate in $(b)$ awaits spectroscopic confirmation.

nucleus itself were measured by fitting two-dimensional elliptical Gaussians to the 1 ."3 resolution image. Results are given in Table 3.

In the $\mathrm{S}$ nebula we identify tentatively the embedded bright knot as a Mach disk of the active outflow, while the arcuate feature is a good candidate for a bow shock. These identifications are justified in $\S 4$. The narrow dimension in the HST images of both nebulae is unresolved at the resolution of the interleaved WFC frames (in which we estimate from stars that 0.15 diameter encircles $80 \%$ energy at the wavelength of our images). The curvature of the $\mathrm{N}$ hot spot nebula is less than that in the S, but our spectra detailed in the next section support its interpretation as an oblique shock. Like the $\mathrm{S}$ feature, its line emission is on the leading edge of the radio hot spot and it shows large velocity shifts relative to the adjacent ISM.

Beyond the $\mathrm{N}$ and $\mathrm{S}$ ionized nebulae, there is one other prominent jet/ISM interaction. A "ridge" extending for $\approx 8^{\prime \prime}$ along the NW anomalous arm, is bright at radio (Fig. 4) and X-ray (CWD) frequencies, and is labeled "Oblique shock?" in Figure 4. In this figure, a sharp-edged, dusty nebula is located just before the anomalous arms bifurcate. The sharp edge lies along the anomalous arms, and embedded dust blocks background line emission.

\subsection{Overview of Kinematic Patterns and Energetics of the High-Velocity Gas}

The [O III] $\lambda 5007$ luminosity is correlated with $1.5 \mathrm{GHz}$ radio power in Seyfert galaxies (e.g., Whittle 1985). Bicknell et al. (1998) have summarized this correlation recently for the full zoo of radioactive galaxies. Deriving unreddened fluxes of the hot spots, we find that both hot spots in NGC 4258 plot on their correlation.

The emission line profiles across the two hot spot nebulae are double-peaked and very broad. Both nebulae show pronounced kinematic curvature along the slit and spatial cur- 
TABLE 3

Characteristics of the Radio Sources at $1.5 \mathrm{GHz}$

\begin{tabular}{cccccc}
\hline \hline & \multicolumn{2}{c}{ Nuclear Distance $(\operatorname{arcsec})$} & Fitted Source Size \\
\cline { 2 - 5 } Source & Optical & Radio & $\begin{array}{c}\text { Flux Density } \\
(\mathrm{mJy})\end{array}$ & $\begin{array}{c}\text { Minimum Pressure } \\
\left(\text { dyn } \mathrm{cm}^{-2}\right)\end{array}$ \\
\hline Nucleus ......... & $\ldots$ & $\ldots$ & $\ldots$ & $11.7 \pm 0.5$ & $\ldots$ \\
N hot spot ...... & $49.6 \pm 0.1$ & $49.2 \pm 0.25$ & $14 \times 12$ & $15 \pm 2$ & $1.8 \times 10^{-11}$ \\
S hot spot ...... & $25.0 \pm 0.1$ & $24.5 \pm 0.25$ & $22 \times 18$ & $32 \pm 2$ & $1.2 \times 10^{-11}$ \\
\hline
\end{tabular}

NoTE.- Integrated flux density and nuclear distances of the radio features are obtained from two-dimensional Gaussian fits to the sources in the 1".3 resolution image. The distances for the optical sources are those of the flux peaks. The last column is derived from the radio observations (see, e.g., Leahy 1991).

vature on the sky. But, whereas the low-velocity component of line profiles near the $\mathrm{S}$ nebula has fixed velocity along its length, that component of the $\mathrm{N}$ nebula shows a substantial velocity gradient. These patterns constrain the kinematic models to be discussed in $\S 4.1$.

Table 4 summarizes the energetics of other high-velocity features. East of the $\mathrm{S}$ nebula is a partial Doppler ellipsoid that we will discuss in $\S 3.6 .2$. Its kinematics resembles those of the S nebula. In the N spectrum (Fig. 8) there is a clear break in the velocity field SW of the nebula, when the slit crosses one of the anomalous arms.

\subsection{Emission Line Properties}

Figure 9 shows spectra summed across three regions of Figure $7 b$ along the long-slit spectrum that intersects the $\mathrm{S}$ nebula and its putative Mach disk. We used the interactive deblend option in the IRAF task "splot" to fit Gaussians to the line profiles. Each profile along the slit was fitted with a narrow (2-3 pixels) and wide (6-8 pixels) Gaussian pair, with velocity separation constant for all lines. The [N II] and [O III] doublet flux ratios were fixed at their expected values. The $[\mathrm{S}$ II $]$ doublet emission was found to always be in its low-density limit $\left(n_{e} \lesssim 100 \mathrm{~cm}^{-3}\right)$.

We compared fluxes from our long-slit spectra with those from the continuum-subtracted WFPC2 image to verify our flux procedures. We summed counts from the WFPC2 image of the $\mathrm{S}$ hot spot nebula, scaled them by 0.55 to correct for the $\mathrm{H} \alpha$ contribution in the HST filter (Fig. 12), and found that the $\mathrm{S}$ nebula has a total observed [N II] $\lambda 6583$ flux of $5 \pm 1 \times 10^{-15} \mathrm{ergs} \mathrm{s}^{-1} \mathrm{~cm}^{-2}$; this is $50 \%$ larger than the spatial sum of this feature from the long-slit spectra and consistent with the fraction excluded by the slits.

Figure 11 shows our fits to spectra of the N nebula; the blue component is broad, so is a shock candidate. Line fluxes and excitation ratios derived from these fits are summarized in Table 5. Figure 12 shows that the WFPC 2 filter F658N transmits little $\mathrm{H} \alpha$ from the blueshifted component of the $\mathrm{N}$ hot spot nebula; the observed flux in this filter comes in roughly equal parts from the $\mathrm{H} \alpha$ low-velocity and [N II] $\lambda 6583$ low- and high-velocity components. The longslit spectrum confirms the WFPC2 result that the N nebula has $[\mathrm{N} \mathrm{II}] \lambda 6583$ flux $3.1 \pm 0.5 \times 10^{-15} \mathrm{ergs} \mathrm{s}^{-1} \mathrm{~cm}^{-2}$; essentially the entire structure was included in our long-slit spectra.

We used the observed $\mathrm{H} \alpha / \mathrm{H} \beta$ flux ratios to determine average reddening toward both nebulae. Assuming that the intrinsic flux ratio $\mathrm{H} \alpha / \mathrm{H} \beta=2.94 \pm 0.08$ (Dopita \& Sutherland 1995, hereafter DS) and that the extinction is a foreground screen and therefore is unmixed with the high-velocity gas, we used our line fits to find that $A_{V}=1.7$ \pm 0.1 mag across the west end of the $S$ feature, $0.75 \pm 0.05$ to the east, and $A_{V}=1.0 \pm 0.1 \mathrm{mag}$ across the $\mathrm{N}$ feature.

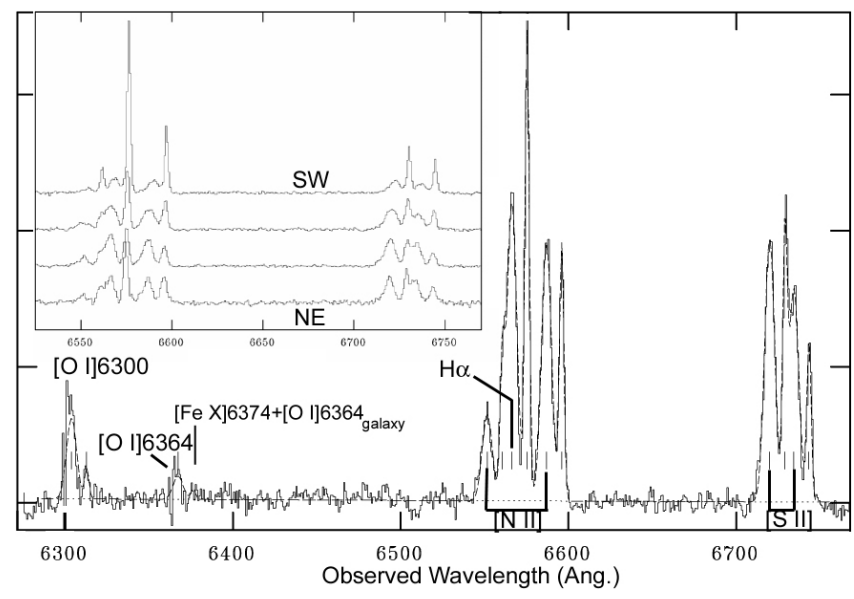

Fig. 11.- Spectrum of the $\mathrm{N}$ hot spot nebula averaged between the horizontal ticks in Fig. 8, together with Gaussian fits (inset shows the spatial variation of the $[\mathrm{N} \mathrm{II}]+\mathrm{H} \alpha$ and $[\mathrm{S} \mathrm{II}]$ complexes); the blueshifted broad component ( $300 \mathrm{~km} \mathrm{~s}^{-1}$ FWHM) on each emission line which arises from the main shock is labeled by its transition while the corresponding redshifted galaxy line is not. The position where shocked [Fe $\mathrm{x}]$ $\lambda 6374$ (see Wilson \& Raymond 1999) would appear is also marked; any emission is blended with an [O I] transition from the galaxy ISM whose flux is set by the other transition in the doublet to a value that excludes significant shocked $[\mathrm{Fe} \mathrm{x}]$ flux.

TABLE 4

Derived Physical Properties of the High-Velocity Gaseous Features

\begin{tabular}{|c|c|c|c|c|}
\hline Feature & $L_{\mathrm{H} \alpha}\left(\times 10^{38} \operatorname{ergs~s}^{-1}\right)$ & $n_{0}\left(f^{-1 / 2} \mathrm{~cm}^{-3}\right)^{\mathrm{a}}$ & $\operatorname{Mass}\left(\sqrt{f} M_{\odot}\right)$ & $\operatorname{KE}(\sqrt{f}$ ergs $)$ \\
\hline $\mathrm{N}$ shock & $0.46 \pm 0.15$ & $1.3 \pm 0.3$ & $\ldots$ & $\ldots$ \\
\hline$\ldots \ldots \ldots \ldots$ & $1.1 \pm 0.3$ & $1.6 \pm 0.3$ & $\cdots$ & $\ldots$ \\
\hline S Mach disk ......... & $0.16 \pm 0.03$ & $40 \pm 5$ & $10^{3}$ & $\ldots$ \\
\hline Nuclear "Loop"...... & $9.4 \pm 0.4$ & $18 \pm 4$ & $1.6 \times 10^{5}$ & $2.5 \times 10^{52}$ \\
\hline Bubble $1 \ldots \ldots \ldots \ldots$ & $5.0 \pm 0.7$ & 2.4 & $6.9 \times 10^{5}$ & $1.1 \times 10^{53}$ \\
\hline
\end{tabular}

${ }^{a}$ Uncertainties in derived densities should be propagated to the other columns. 


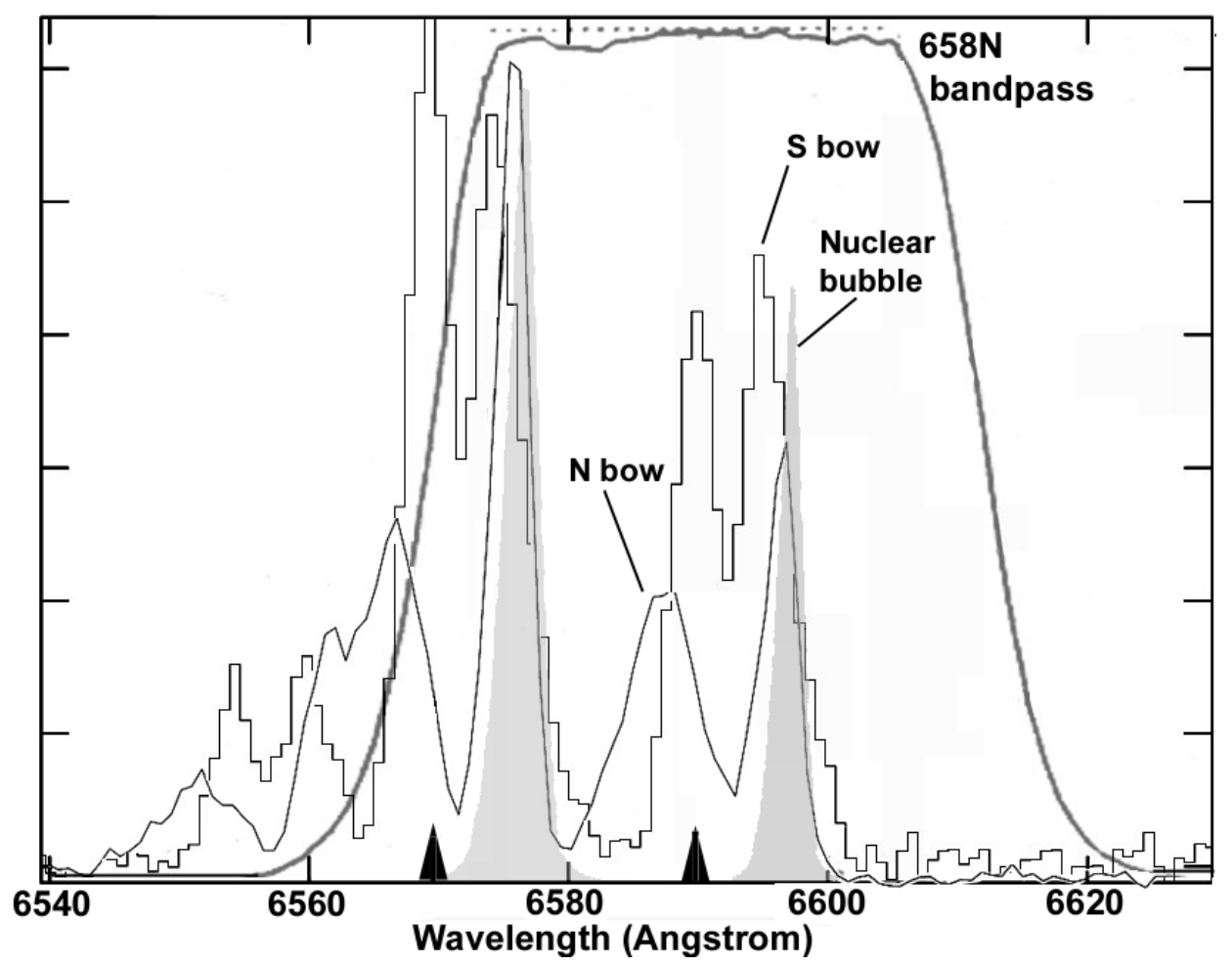

FIG. 12. - The F658N filter efficiency (see WFPC2 Instrument Handbook, Third ed, Appendix 8.2.6) is plotted on the spatial average of the (histogram) S and (solid) $\mathrm{N}$ hot spot nebula's [N II] $\lambda \lambda 6548,6583+\mathrm{H} \alpha$ complex; the average spectrum of the "Nuclear Loop" bubble is also shown in gray. The filter passes [N II] $\lambda 6583$ at total efficiency QT $=0.102$ while $\mathrm{H} \alpha$ is passed with QT $\approx(0.08,0.01,0.10)$ for the $(\mathrm{S}, \mathrm{N})$ nebulae and "Nuclear Loop" bubble, respectively. Arrowheads at bottom mark the galaxy systemic velocity in the $\mathrm{H} \alpha($ left) and [N II] $\lambda 6583$ (right) lines.

Correcting for reddening, we obtained $\mathrm{H} \alpha$ luminosities of $1.1 \pm 0.3 \times 10^{38}$ and $4.6 \pm 1.5 \times 10^{37} \mathrm{ergs} \mathrm{s}^{-1}$ for the highvelocity gas in the west end of the $\mathrm{S}$ nebula and whole $\mathrm{N}$ nebula, respectively, and $3.7 \times 10^{38} \mathrm{ergs} \mathrm{s}^{-1}$ for the entire $\mathrm{S}$ complex. For comparison, CMV found $A_{V} \approx 1$ on average along most of the SE anomalous arm, and the total $\mathrm{H} \alpha$ luminosity of the anomalous arms is $9.3 \pm 2.9 \times 10^{39}$ $\mathrm{ergs} \mathrm{s}^{-1}$ (CWD, corrected to $7.2 \mathrm{Mpc}$ distance. Internal reddening is the biggest source of uncertainty).

\subsection{Gaseous Kinematics 3.6.1. Shock Signatures}

Abrupt, large kinematic disturbances indicate that the high-velocity emission results from shocks, so we henceforth refer to the nebulae as the $\mathrm{N}$ and $\mathrm{S}$ shock complexes. Except for [O III] $\lambda 5007$, all emission lines exhibit the same spatio-kinematic variations. In this section we examine gas motions at different positions along the slits.

We first establish the recessional velocity of the rest frame of the ISM ahead of each shock. CWT used the HIFI FP spectrometer to map ionized gas motions across NGC 4258. Because of its large inclination angle, the rotating disk could be measured by CWT out to $6^{\prime}(13 \mathrm{kpc})$ galactocentric radius. The best-fit model of the motions, summarized in their Figure $9 b$, includes the influence of a weak stellar bar or oval distortion with major axis along P.A. $\sim 15^{\circ} \pm 2^{\circ}$. This model has gas on bar-forced orbits out to well beyond the radii of the hot spot nebulae. The bar-forced velocities are marked along our long-slit spectrum in Figure $7 a$.

According to the CWT model, gas in the galaxy disk near the $S$ bow shock should be moving at $410 \pm 20 \mathrm{~km} \mathrm{~s}^{-1}$ heliocentric. Near the $\mathrm{N}$ and $\mathrm{S}$ shock complexes, these are the velocities of the flux minimum between the two peaks of each emission line profile in Figure 7. There is no evidence from the $\mathrm{H} \beta / \mathrm{H} \alpha$ flux ratio at this velocity that the minimum is due to dust extinction; it looks instead like gas is deficient at these velocities. Our HST WFPC2 image shows diffuse emission near the $\mathrm{S}$ shock (filling the insert image at the bottom of Fig. 5). The long-slit spectra (Fig. 7b) show that most of this emission corresponds to the narrow "I" (blue) part of the "IC"-shaped emission line profiles, which is blueshifted by $-160 \mathrm{~km} \mathrm{~s}^{-1}$ relative to the galaxy disk. The high-velocity prongs corresponding to the tips of the " $\mathrm{C}$ " in Figure 7 are redshifted by $\$ 410 \mathrm{~km} \mathrm{~s}^{-1}$ from the local disk velocity; therefore, all emission from the $S$ shock is redshifted relative to the local galactic disk. This pattern constrains the models to be discussed in $\S 4.1$.

Near the N shock, CWT find velocities of $510 \pm 40$ $\mathrm{km} \mathrm{s}^{-1}$ heliocentric. This is $-65 \mathrm{~km} \mathrm{~s}^{-1}$ blueshifted from the narrower (red) peak of the double-peaked profiles of the $\mathrm{N}$ shock complex. All of the emission from the $N$ shock is blueshifted from the ambient disk by -327 (peak) to -687 (blue limit) $\mathrm{km} \mathrm{s}^{-1}$. Along the slit, both components of the $\mathrm{N}$ shock follow the velocity field of the galaxy disk.

\subsection{2. "Bubble" Extension of the $S$ Shock}

The "Doppler ellipsoid" $\mathrm{E}$ of the $\mathrm{S}$ shocked nebula, labeled " 1 " in Figures 10 and 7, with diameter 7". $6=265$ pc, shares the kinematics and excitation patterns of the $S$ bow shock, so is likely to be part of the larger shock complex. If a hemisphere, then our two slits include $80 \%$ of its area projected on the sky. The average of a 2".5 wide spectral slice through the bubble shows line splitting of 315 $\mathrm{km} \mathrm{s}^{-1}$. Correcting for instrumental resolution in quadrature, the redshifted knot has FWHM $165 \mathrm{~km} \mathrm{~s}^{-1}$, while the 
TABLE 5

Spectral Parameters for the Blue and Red Emission Line Components

\begin{tabular}{|c|c|c|c|c|c|c|}
\hline \multirow[b]{2}{*}{ TRANSITION } & \multicolumn{3}{|c|}{$F\left(\times 10^{-16} \operatorname{ergs~s}^{-1} \mathrm{~cm}^{-2}\right)^{\mathrm{a}}$} & \multicolumn{3}{|c|}{ DEREDDENED $F / F(\mathrm{H} \alpha)^{\mathbf{b}}$} \\
\hline & $\begin{array}{l}\text { Mach Disk } \\
\text { S Nebula }\end{array}$ & $\begin{array}{l}\text { W End of } \\
\text { S Nebula }\end{array}$ & $\begin{array}{c}\mathrm{N} \\
\text { Nebula }\end{array}$ & $\begin{array}{l}\text { Mach Disk } \\
\text { S Nebula }\end{array}$ & $\begin{array}{l}\text { W End of } \\
\mathrm{S} \text { Nebula }\end{array}$ & $\begin{array}{c}\mathrm{N} \\
\text { Nebula }\end{array}$ \\
\hline $\mathrm{H} \beta^{*} 2.94, B \ldots \ldots$ & 0.5 & 1.7 & $11.5^{\mathrm{c}}$ & 1 & 1 & 1 \\
\hline $\mathrm{H} \beta^{*} 2.94, R \ldots \ldots$ & 0.8 & 1.3 & 5.2 & 1 & 1 & $\ldots$ \\
\hline$[\mathrm{O}$ III $], B \ldots \ldots .$. & 2.5 & 2.1 & 16 & 1.5 & 1.2 & 1.3 \\
\hline$[\mathrm{O} \mathrm{III}], R \ldots \ldots .$. & 2.2 & 1.3 & 3.8 & 3.2 & 1.1 & $\ldots$ \\
\hline$\left[\mathrm{O}_{\mathrm{I}}\right], B \ldots \ldots .$. & $\ldots$ & $\ldots$ & 5.1 & $\ldots$ & $\ldots$ & 0.3 \\
\hline$[\mathrm{O} \mathrm{I}], R \ldots \ldots .$. & $\ldots$ & $\ldots$ & $\ldots$ & $\ldots$ & $\ldots$ & $\ldots$ \\
\hline$[\mathrm{N}$ II $], B \ldots \ldots .$. & 1.6 & 1.6 & 19 & 0.8 & 0.9 & 1.2 \\
\hline$[\mathrm{N}$ II $], R \ldots \ldots .$. & 1.1 & 1.8 & 8.9 & 1 & 0.9 & $\ldots$ \\
\hline $\mathrm{H} \alpha, B \ldots \ldots \ldots$ & 1.9 & 1.9 & 16 & $\ldots$ & $\ldots$ & $\ldots$ \\
\hline $\mathrm{H} \alpha, R \ldots \ldots \ldots \ldots$ & 1.1 & 1.9 & 17 & $\ldots$ & $\ldots$ & $\ldots$ \\
\hline$[\mathrm{S}$ II $], B \ldots \ldots .$. & 2.1 & 2.5 & 26 & 0.9 & 1.3 & 1.6 \\
\hline \multirow[t]{3}{*}[\mathrm{S}\text{II}]{,$R \ldots \ldots .}$. & 1.6 & 2.9 & 8.3 & 1.4 & 1.5 & $\ldots$ \\
\hline & \multicolumn{3}{|c|}{ FWHM $\left(\mathrm{km} \mathrm{s}^{-1}\right)^{\mathrm{d}}$} & \multicolumn{3}{|c|}{$A_{V}$} \\
\hline & $\begin{array}{c}\text { Mach Disk } \\
\text { S Nebula }\end{array}$ & $\begin{array}{l}\text { W End of } \\
\text { S Nebula }\end{array}$ & $\begin{array}{c}\mathrm{N} \\
\text { Nebula }\end{array}$ & $\begin{array}{c}\text { Mach Disk } \\
\text { S Nebula }\end{array}$ & $\begin{array}{l}\text { W End of } \\
\text { S Nebula }\end{array}$ & $\begin{array}{c}\mathrm{N} \\
\text { Nebula }\end{array}$ \\
\hline$B$ (blue) ........... & 90 & 90 & 190 & 0.55 & 0.50 & $1.0 \pm 0.1$ \\
\hline$R($ red) $\ldots \ldots \ldots \ldots$ & 175 & 140 & 80 & $1.6 \pm 0.1$ & $1.8 \pm 0.1$ & $\ldots$ \\
\hline
\end{tabular}

a Observed line fluxes derived from two-Gaussian fits to line profiles in Fig. 9, and for the sum of the $\mathrm{N}$ shock complex. Line doublets are summed where appropriate. Measurement uncertainties are order $10 \%$ on bright lines and $25 \%$ on fainter lines.

${ }^{\mathrm{b}}$ Line flux ratios relative to $\mathrm{H} \alpha$, dereddened using the assumed intrinsic decrement $\mathrm{H} \beta / \mathrm{H} \alpha=0.34$ (DS) and standard Galactic reddening curve of Mathis with $R=3.1$. Uncertainties from the flux measurement are combined with uncertainty from dereddening.

${ }^{c} \mathrm{H} \beta$ line flux was increased by $25 \%$ to correct for Balmer absorption; this may be an underestimate, making dereddening suspect.

${ }^{d}$ Corrected for instrumental resolution in quadrature. Uncertainties are roughly $15 \%$.

blueshifted side is $150 \mathrm{~km} \mathrm{~s}^{-1}$ FWHM. The velocity deviation from galaxy rotation (dashed line in Fig. 7a) is larger for the blue than red component. The HST image (Fig. 10) shows a faint arcuate feature in this region, supporting a shock interpretation. Summing the observed $\mathrm{H} \alpha$ fluxes of the bubble in our two slits, we obtained $2 \pm 0.25 \times 10^{-14}$ ergs $\mathrm{s}^{-1} \mathrm{~cm}^{-2}$. Both velocity systems in the bubble are reddened like the low-velocity component near the $\mathrm{S}$ shock, $A_{V}=0.75 \pm 0.05$. To deredden and account for the unobserved flux, we therefore increased the observed flux $1.76 \pm 0.07 \times 1.25$-fold to find $L_{H \alpha}=2.5 \pm 0.5 \times 10^{38}$ ergs s$~^{-1}$ from volume $1.4 \times 10^{62} \mathrm{~cm}^{3}$. Following the procedure in $\S 3.1$, we derived an rms gas density $2.4 \pm 0.2 f^{-1 / 2}$ $\mathrm{cm}^{-3}$, ionized mass $M_{\text {ion }}=3.5 \pm 0.4 \times 10^{5} \sqrt{f} M_{\odot}$, hence kinetic energy $\gtrsim 5 \times 10^{52} \sqrt{f}$ ergs. The last requires e.g., $\gtrsim 50 \sqrt{f}$ supernovae. If we interpret line widths as turbulent velocities, then the bubble walls have a comparable turbulent energy. ${ }^{7}$

We made the approximations that energy is injected at a constant rate during the bubble's evolution, that the shell shock is strong so that we can ignore the thermal pressure of the ambient gas, and that the bubble is smaller than the scale height of the galaxy gas disk so the ambient ISM density is constant. Then in the energy conserving phase of the bubble interior, the bubble radius and expansion velocity are related: $R=1.67 v t$. From the observed values,

\footnotetext{
${ }^{7}$ Note that the apparent counter-feature to this bubble $30^{\prime \prime} \mathrm{NW}$ of the nucleus that is visible as broad emission line profiles in Figs. $5 a$ and $5 e$ of CMV is an artifact of a reflective ghost in the FP spectrometer used to make those images.
}

$t=0.52 \mathrm{Myr}$. The energy injection rate $L$ follows from $R(t)=31\left(L_{36} / n_{0}\right)^{0.2} t_{6}^{0.6} \mathrm{pc}=133 \mathrm{pc}$ in $t_{6}=0.52\left(\right.$ where $L_{36}$ has units $10^{36} \mathrm{ergs} \mathrm{s}^{-1}$ ), so $L=10^{6} \sqrt{f} L_{\odot}$.

The maximum velocities reduce smoothly away from the $\mathrm{S}$ shock and are resolved spatially as a red wing that extends E of the S shock (labeled " 2 " in Fig. 7a), suggesting that bubble and $\mathrm{S}$ shock are connected dynamically. In scale and energies (kinetic and turbulent) this bubble resembles the N bubble in the NLR of M51 (Cecil 1988), likely a jet-generated structure.

\subsection{3. "Scalloped" Velocities Across Strands of the SE Anomalous Arm}

There are "scalloped," arcuate features farther along the S long-slit spectra, in the NE quadrant of the galaxy. Rubin \& Graham (1990) called them a "drizzle" onto the galaxy disk. Each occurs when the slit crosses one of the arcuate strands of the anomalous arms and has a symmetric kinematic shift across each strand from redshift to systemic velocity back to redshift for a maximum velocity excursion of $50 \mathrm{~km} \mathrm{~s}^{-1}$. This shift is comparable to the amplitude of the kinematic wiggles seen in the FP spectra at radii of more than 50" (Fig. 11 of CWT) along the three main strands that form the SE anomalous arm.

\subsection{4. "Nuclear Loop" Bubble}

This is the bright "?" shaped feature prominent in the PC frame (Fig. 5). CWT showed (their Fig. 17a) that it lies along the kinematic line of nodes but is redshifted by 125 $\mathrm{km} \mathrm{s}^{-1}$ from the adjacent ISM, indicating that it is not in rotation. The PC image shows that the arc is actually a full ring (the "Nuclear Loop" bubble). Its E half is half as bright 
as the $\mathrm{W}$, but there is no evidence from the color map (Fig. $6 b$ ) of enhanced reddening. In $\S 4.3 .2$ we present an alternative explanation for its relative faintness.

We obtained an $\mathrm{H} \alpha$ flux of $1.6 \pm 0.1 \times 10^{-13}$ ergs s ${ }^{-1} \mathrm{~cm}^{-2}$ for the ring. Assuming no reddening, $L_{\mathrm{H} \alpha} \gtrsim$ $9.6 \pm 0.4 \times 10^{38} \mathrm{ergs} \mathrm{s}^{-1}$. We assumed that the bubble is toroidal with thickness $50 \mathrm{pc}$ and overall diameter 5".6 = 200 pc. Following the analysis of $\S 3.1$, and assuming that the whole torus of volume $9 \times 10^{60} \mathrm{~cm}^{3}$ expands through the ISM at $125 \mathrm{~km} \mathrm{~s}^{-1}$, we derived a gas density, ionized mass, and kinetic energy of $\gtrsim 18 f^{-1 / 2} \mathrm{~cm}^{-3}, \gtrsim 1.6$ $\times 10^{5} \sqrt{f} M_{\odot}$, and $\gtrsim 2.5 \times 10^{52} \sqrt{f}$ ergs, respectively.

3.6.5. Features Associated with Bar Shocks

There is an $\approx 130 \mathrm{~km} \mathrm{~s}^{-1}$ (projected) velocity jump $\sim 16^{\prime \prime}$ $\mathrm{W}$ of the bow shock along the slit. There is also an $\approx 40$ $\mathrm{km} \mathrm{s}^{-1}$ (projected) velocity jump $\sim 17^{\prime \prime} \mathrm{NE}$ along the slit from the $\mathrm{N}$ shock complex. Both are consistent with shocks along the leading edges of the putative bar at P.A. $=15^{\circ} \pm 2^{\circ}$ if the bar rotates in the same sense as the rest of the galaxy. In fact, the $\mathrm{S}$ nebula is clearly just in front of the dust lane. We discuss bar shocks in $\S 5.4$.

\section{DISCUSSION}

In this section we constrain properties of the two shock complexes and the properties of the putative Mach disk, using the spatio-kinematic patterns in the long-slit ISIS spectra that were discussed in $\S$ 3.6.1. We show that the patterns evident in the shocked, $\mathrm{S}$ hot spot nebula are consistent with a bow shock, while the $\mathrm{N}$ hot spot nebula has the characteristics of an oblique shock. We consider if the shocked structures are photoionized by the AGN.

\subsection{Modeled Properties of the Shocks 4.1.1. Model Assumptions}

We used the kinematical model of Hartigan, Raymond, \& Hartmann (1987, hereafter HRH) and Hartigan, Raymond, \& Meaburn (1990). This model stitches together radiative, planar shocks along the surface of the bow shape $z(r)=\alpha r^{2}+\alpha^{3} \beta r^{4}(\alpha$ and $\beta$ are fitted parameters) where $z$ and $r$ are measured along the axis of symmetry and perpendicular to it, respectively. ${ }^{8}$ Raga $\&$ Bohm (1986) show that this shape arises in postshock ionization equilibrium near the shock apex when the generating object is spherical. Assuming that the outflow axis of the $\mathrm{S}$ bow shock is inclined $\phi \approx 30^{\circ}$ to our line of sight (to be justified in $\S$ 4.1.2), we fit the observed shape when $\alpha=0.035$ and $\beta=20$.

Our models assumed that the postshock cooling distance is small, so that all radiation originates in a thin shell. This assumption is valid over most of the bow, except near its apex. However, at the velocities across the $\mathrm{N}$ and $\mathrm{S}$ shock complexes, $V_{s}$ is high enough for the hot postshock gas to generate ionizing radiation even away from the apex. Models do not consider this local radiation (Taylor, Dyson, \& Axon 1992; Ferruit et al. 1997, 1999) and do not mix particles of different temperature. Our particular models did not include radiation from the AGN.

\footnotetext{
${ }^{8}$ We previously used these models to generate full three-dimensional "data cubes" to interpret imaging spectrophotometry of Herbig-Haro objects. The present observations are constrained by slit sampling. The case when the entire bow shock lies in the slit is incorporated in the HRH models. Here we have only two slices along the bow, and $\approx 4$ resolution elements across.
}

$V_{s}$ and orientation angle $\phi$ between the shock axis and our line of sight are the most important parameters for comparing the line profiles of bow-shock models with the observed spectra. HRH show that $V_{s}$ equals the full width at zero intensity (FWZI) for radiating bow shocks, independent of orientation angle, preshock density, bow-shock shape, and preshock ionization state. On this basis, from Figure 7 we found $V_{s} \approx 400-450 \mathrm{~km} \mathrm{~s}^{-1}$ for the $\mathrm{S}$ bow shock.

No published models slice bow shocks with comparable resolution and sampling to our ISIS spectra. (They average across in $r$ but resolve in $z(r)$, opposite to our spectra.) To maximize the diagnostic capabilities of our data, we have therefore generated exploratory model "data cubes" with the HRH bow-shock code, ${ }^{9}$ revised to use planar shock emission line grids from the MAPPINGS II code (DS). The MAPPINGS models span $V_{s}=150-500 \mathrm{~km} \mathrm{~s}^{-1}$ at small enough velocity increments to cover the shape of the $S$ bow shock. We smoothed, rotated, and sliced them spatially to mimic our spectra, see Figure 13.

\subsubsection{Orientation and Flow Pattern of the S Bow Shock}

Previous spectroscopy has placed the anomalous arms close to the galaxy disk. Arguments favoring this orientation include the following: gas velocities consistent with oval-forced motions within the disk (CWT), X-rays from entrained hot gas, optical emission line ratios, and kinematic discontinuities which are consistent with shocks of several hundred $\mathrm{km} \mathrm{s}^{-1}$ that flow into an ISM with density too large to be found outside the galaxy disk (CWD; CMV). In this section we constrain the orientation of the active flow. We show that the kinematics and extinction pattern of the $\mathrm{S}$ bow shock is consistent with flow near the galactic disk.

\subsubsection{Propagating Shocks}

Figure 13 (left) shows model data cubes of the velocity field generated by a bow shock that is presented to us at a full range of viewing angles $\phi$, then sliced with slits oriented like our spectra. The top three panels show bow shocks around invisible "bullets" that are moving into a stationary ISM at $350 \mathrm{~km} \mathrm{~s}^{-1}$; none have kinematic structure that agrees with our data. In particular, if the bow shock propagates along the spin axis of the accretion disk, it would be moving almost $90^{\circ}$ to us, and so would produce a symmetric "Doppler ellipsoid" centered on the rest velocity of the ambient ISM (top left of Fig. 13). This is not the observed pattern.

\subsubsection{Stalled Shock}

The bottom panel in Figure 13 shows a bow shock at rest while the ambient ISM flows at $350 \mathrm{~km} \mathrm{~s}^{-1}$ from left to right, opening the bow shock in the downstream direction. To match the observed spatio-kinematic pattern we must reverse the spatial orientation of this model shock and shift its mean velocity. This is accomplished in a backflow perhaps after outflow splashes against an obstacle. Figure 13 (right) shows space velocity plots that simulate long-slits to mimic the ISIS placements on the S bow shock; evidently, the models reproduce the main observed spatiokinematic patterns. The high-velocity $\mathrm{C}$-shaped emission in our space velocity plot would come from the backflow past the stalled shock.

\footnotetext{
${ }^{9}$ We thank J. Morse for providing us with the original code written by P. Hartigan, which we have modified.
} 


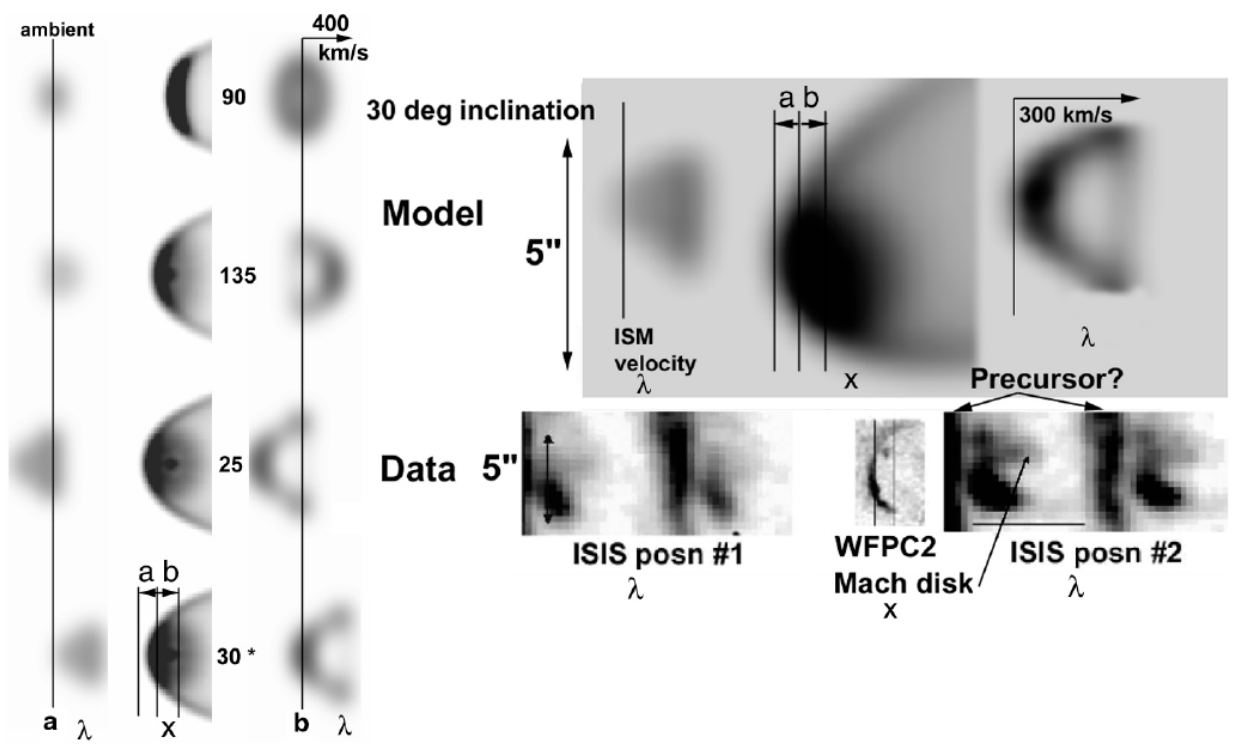

FIG. 13.-Model bow shocks compared to our long-slit spectra. Emission from the Mach disk and the photoionized shock precursor are not modeled. Vertical axes are always spatial while the horizontal axes are either spatial (" $X$ " below each column) or velocity (" $\lambda$ "). The resolution of the spatial image is that of our HST image of the S bow shock, while the velocity images are sampled and smoothed like our long-slit WHT data. The vertical lines show the velocity of the fully preionized preshock ISM. (Left) Model bow shocks moving at $V_{s}=350 \mathrm{~km} \mathrm{~s}^{-1}$ in the ISM, labeled by their inclination angle $\phi$. Only the model in the bottom panel has the spatio-kinematic distribution of our data across the S shock complex. In that panel, ISM flows left to right onto a stationary cloudlet, as would occur, e.g., in a backflow around a stalled jet head. (Right) Example model bow shock in $\mathrm{H} \alpha$ emission for $V_{s} \approx 350 \mathrm{~km} \mathrm{~s}{ }^{-1}$, inclined $\phi=30^{\circ}$ to our sight line, rotated and smoothed to the resolution of the ISIS spectra. Left and right top panels show space-velocity plots of H $\alpha$; zero velocity is at left. The gas backflows. The model compares favorably to the " $\mathrm{C}$ " part of the observed "IC" pattern in the bottom panels.

The good agreement with data suggests that a bow shape that refracts and accelerates ISM flowing with low turbulence captures the essential dynamics of the $\mathrm{S}$ shock complex. In this simplified picture the upstream wall might be dense molecular clouds whose outer skins are ionized by impacting jet material. The low-velocity l-shaped emission near the $\mathrm{S}$ bow shock might be this combined ionization/ shock front associated with the radiative precursor.

Backflow or ablata from the working surface of the $S$ jet will entrain ambient gas. The main entrainment shock would be pressure-balanced by a wall shock moving into the ambient ISM. The high-velocity (" $\mathrm{C}$ ") component that we noted in § 3.6.1 is plausibly the entrainment shock, while the low-velocity ("I") emission with absolute deviation from the ambient ISM of $165 \mathrm{~km} \mathrm{~s}^{-1}$ is likely from the wall shock. An ambient ISM more than 10 times denser than the entrained medium produces this shift and is plausible because our models orient the S shock close to the galaxy plane that is rich in molecular gas (Cox \& Downes 1996).

\subsubsection{An Oblique Shock to the $N$}

The $\mathrm{N}$ shock complex appears less obviously bowlike than that to the $S$, so we have not attempted to fit its shape. This necessarily limits what we can say about its orientation hence dynamics. While the spatio-kinematic pattern in Figure 8 resembles that of the $\mathrm{S}$ bow shock, the overall velocity displacement of the whole $\mathrm{N}$ complex relative to the bar-forced galaxy disk is opposite to that of the $\mathrm{S}$ bow shock: the apex of the $\mathrm{N}$ shock shows the largest velocity deviations from ambient while the wings show the smallest deviations. The bow-shock model in the third row of Figure 13 most closely reproduces this pattern. Again this can be interpreted as the signature of a backwash of $\approx 350 \mathrm{~km} \mathrm{~s}^{-1}$ to us. Necessarily this would imply low-velocity redshifted emission from the wall shock and ambient gas near the $\mathrm{N}$ shock deviates by $\approx 65 \mathrm{~km} \mathrm{~s}^{-1}$ from the motion of the galaxy ISM. However, Figure 8 shows reduced low-velocity emission near the $\mathrm{N}$ shock, so the putative wall shock must be extinguished by the galaxy disk.

\subsubsection{Emission Line Flux Ratios Compared to Shock Models}

To maximize signal-to-noise ratios, we averaged the spectrum near the apex of the S bow shock. In Table 6 we compare the dereddened line ratios of the high-velocity component to the results of various planar shock models computed with the MAPPINGS III code. Flux ratios of the limited lines available in the spectrum are most consistent with "c" models and $V_{s} \approx 300 \mathrm{~km} \mathrm{~s}^{-1}$. For this $V_{s}$, the models of DS predict that the flux ratio [Fe $\mathrm{x}] \lambda 6374 /$ $\mathrm{H} \alpha \approx 0.04$. At the $\mathrm{S}$ shock, this line is blended with [O I] emission from the galaxy ISM, and we would not detect a line fainter than $\approx 2$ times this flux.

DS found that shocks of $500 \mathrm{~km} \mathrm{~s}^{-1}$ generate enough radiation to reproduce the average ionization parameter found in the circumnuclear regions of AGNs. Optical spectra of NGC 4258 (CMV) show LINER-like emission near the nucleus (see also Fig. 11), whose softer spectrum is reproduced by planar shock models (DS) with somewhat lower $V_{s}=300-400 \mathrm{~km} \mathrm{~s}^{-1}$, or by photoionization models (e.g., Shields 1992). We consider ionization ratios in more detail in $\S 4.3$.

\subsubsection{Bow Shock Physical Properties}

The cooling length behind a planar shock $L_{\text {cool }}=$ $11.3 V_{s 300}^{4} / n_{0} \mathrm{pc}$ (units of $300 \mathrm{~km} \mathrm{~s}^{-1}$ and $\mathrm{cm}^{-3}$, respectively; DS). ${ }^{10}$ The $\mathrm{S}$ bow shock is unresolved across its narrow dimension in the WFPC2 image (i.e., $L_{\text {cool }}<6 \mathrm{pc}$ ). To further constrain dynamics, we now estimate $n_{0}$ from the

\footnotetext{
${ }^{10}$ Refinements by Ferruit et al. (1997) (updated cooling rates, noncollisional ionization equilibrium, and complete adiabatic conversion of the normal component of incident momentum) double the estimated cooling length. An ambient magnetic field leads to further growth and uncertainties.
} 
TABLE 6

Dereddened Line Ratios to H $\alpha$ versus Models of Planar, Photoionizing Shocks

\begin{tabular}{|c|c|c|c|c|c|c|}
\hline Transition & $(\mathrm{S}, \mathrm{N})$ Dereddened $F_{\lambda} / F_{\mathrm{H} \alpha}$ & $\mathrm{b} 300^{\mathrm{a}, \mathrm{b}}$ & $\mathrm{b} 450$ & $\mathrm{c} 150^{\circ}$ & c300 & $\mathrm{c} 450$ \\
\hline$[\mathrm{O}$ II] $\lambda 3727$. & $5.1 \pm 1.3^{\mathrm{d}},$. & 1.8 & 2.2 & 2.2 & 3.5 & 4.6 \\
\hline He II $\lambda 4686 \ldots \ldots \ldots \ldots$ & $<0.1,<0.1$ & 0.02 & 0.04 & 0.02 & 0.05 & 0.09 \\
\hline $\mathrm{H} \beta \ldots \ldots \ldots \ldots \ldots \ldots . . . . . . .$. & & 0.33 & 0.34 & 0.33 & 0.34 & 0.34 \\
\hline$[\mathrm{O}$ III $] \lambda 4959+07 \ldots \ldots$ & $1 \pm 0.2,1.3 \pm 0.2$ & 1.2 & 1.2 & 0.6 & 0.5 & 1.1 \\
\hline$[\mathrm{O}$ I $] \lambda 6300+64 \ldots \ldots$. & $0.25 \pm 0.05,0.2 \pm 0.05$ & 0.4 & 0.5 & 0.1 & 0.7 & 1.1 \\
\hline$[\mathrm{N}$ II $] \lambda 6548+83 \ldots \ldots$ & $1.6 \pm 0.1,1.2 \pm 0.1$ & 0.6 & 1.0 & 0.5 & 1.3 & 1.6 \\
\hline$[\mathrm{S}$ II $] \lambda 6717+31 \ldots \ldots$. & $2 \pm 0.1,1.6 \pm 0.1$ & 1.1 & 1.1 & 0.5 & 0.8 & 0.9 \\
\hline
\end{tabular}

${ }^{\text {a }}$ Shock speeds in $\mathrm{km} \mathrm{s}^{-1}$

b " $b$ " models: 10:1 density contrast between two colliding shocks, fragmented postshock flows, optically thin precursors, $n_{0}=1 \mathrm{~cm}^{-3}, B=0$.

c "c" models: single shock with no precursor emission and $n_{0}=1 \mathrm{~cm}^{-3}, B=4 \mu \mathrm{G} /\left(n_{0} / 1 \mathrm{~cm}^{-3}\right)^{1 / 2}$.

${ }^{\mathrm{d}}$ Large reddening uncertainty and strong blending with low-velocity emission.

luminosity of the shocked gas. For the shock plus ionized precursor, DS find that $L(H \beta)_{\text {shock }}=\left[1.05 \times 10^{-4} V_{s 300}^{2.41}\right.$ $\left.+1.21 \times 10^{-4} V_{s 300}^{2.28}\right] A n_{0}$ ergs s$^{-1}$, where $\mathrm{A}$ is the shock area, and the shock velocity $V_{s}$ is in units of $300 \mathrm{~km} \mathrm{~s}^{-1}$. Assuming that the $\mathrm{S}$ bow shock is one quarter of a sphere, $A=\pi\left(3^{\prime \prime}\right)^{2}=3.4 \times 10^{4} \mathrm{pc}^{2}$. Our spectra may separate kinematically the shocked gas from its precursor. Therefore, ignoring the second bracketed term and using the previously derived shock-only luminosities, we obtained $n_{0} \approx$ $2.6 \mathrm{~cm}^{-3}$. This density is plausible near molecular clouds in the disk of NGC 4258, hence the $S$ bow gas may derive its entire luminosity from the shock. The $\mathrm{N}$ shock has comparable area and luminosity, so also may be self-luminous.

If this is the case, then $\approx 2 \%$ of the mechanical energy flux is being converted to [O III] $\lambda 5007$ emission and $\approx 0.9 / V_{s 300}^{0.59} \%$ is being converted to $\mathrm{H} \alpha(\mathrm{DS})$. For $350 \mathrm{~km}$ $\mathrm{s}^{-1} \mathrm{~S}$ and $\mathrm{N}$ shocks, we therefore obtain in this picture that the total energy flux $L_{\text {mech }} \approx L_{H \alpha} / 8.2 \times 10^{-3}=1.3 \pm 0.4$ $\times 10^{40}$ and $5.6 \pm 1.8 \times 10^{39} \mathrm{ergs} \mathrm{s}^{-1}$, respectively, from the high-velocity gas alone.

Equipartition pressures for the two radio hot spots were given in Table 3 , and average $\approx 1.5 \pm 0.5 \mathrm{dyn} \mathrm{cm}^{-2}$. To drive a shock at $V_{s}$ into an ambient ISM of density $\rho_{0}$, we need gas pressure $\rho_{0} V_{s}^{2} \approx 4 \times 10^{-9} n_{0} V_{s 400}^{2}$. For the values of $n_{0}$ estimated above, equipartition pressures fail to balance this by a factor of 200, a shortfall typical of Seyfert galaxy jets (Bicknell et al. 1998) that indicates a significant thermal component in the hot spots, or that the lobe particles and fields are out of equipartition, or both. The resulting shock force is $p_{s} A_{s} \approx \rho_{s} V_{s}^{2} \approx 4.5 \times 10^{-6}$ $V_{3}^{-0.4} L_{\mathrm{H} \alpha}$ (Bicknell et al. 1998), which for the $\mathrm{S}$ shock is $7.6 \times 10^{32}$ dyn.

\subsubsection{Shock Precursor}

Photons from hot, postshock gas and from the galaxy AGN (see § 4.3) completely photoionize the preshock ISM to form a radiative precursor if $V_{s}>130 \mathrm{~km} \mathrm{~s}^{-1}$. To estimate the preshock density $n_{0}$ from the assumed precursor emission, we summed several rows of our $\mathrm{N}$ and $\mathrm{S}$ long-slit spectra just below the shock complexes in Figures 7 and 8 . We dereddened the $\mathrm{H} \alpha$ flux from this region by increasing the observed flux by 2.1 times and 1.7 times to obtain $L_{\mathrm{H} \alpha}=6.2 \times 10^{36}$ and $2.9 \times 10^{36} \mathrm{ergs} \mathrm{s}^{-1} \mathrm{arcsec}^{-2}$ from the preshock ISM for $\mathrm{N}$ and $\mathrm{S}$ shock complexes, respectively. Using the formulae in $\S 3.1$, and assuming that the galaxy gas disk has thickness comparable to the lateral extent of the shock $(150 \mathrm{pc})$, we estimate that $n_{0} \approx 1.3 \pm 0.3$ $f^{-1 / 2} \mathrm{~cm}^{-3}$, an upper limit because some of the observed low-velocity flux may come from the wall shock discussed previously. A not very interesting but independent constraint on $f$ comes from the [S II] red-doublet ratios, which are always found in their low-density limit, $n_{e} \lesssim 100 \mathrm{~cm}^{-3}$. Therefore, $f>5 \times 10^{-4}$. A $400 \mathrm{~km} \mathrm{~s}^{-1}$ shock should compress the $[\mathrm{S}$ II] emission zone $\approx 50$-fold (DS), which does not violate the observed low-density limit $(\S 3.5)$ even if $f=1$.

DS show that the radiative precursor should have extent $610 / n_{0} \mathrm{pc}$. The low-velocity component of the emission line profiles near the $\mathrm{S}$ bow shock (bottom right panels of Fig. 13) may come from the precursor as well as the putative wall shock. It is blueshifted from the putative backflowing gas, and our HST line image shows (bottom insert in Fig. 5) that this gas extends diffusely for more than $100 \mathrm{pc}$ across that bow shock.

More evidence that some of the low-velocity gas is the precursor is that the [O III] $\lambda 5007$ profiles differ significantly from those of the other lines. For a $400 \mathrm{~km} \mathrm{~s}^{-1}$ planar shock, $80 \%$ of the [O III] $\lambda 5007$ flux comes from the ionization front (the precursor). In contrast, [S II] and $[\mathrm{N} \mathrm{II}]$ are emitted predominantly from the kinematically more disturbed shock zone. Bow-shock and planar-shock cases differ somewhat. However, Figure 7 shows that [O III] emits more flux proportionally at blueshifted velocities than do the other lines, while the spectrum in the bottom row of Figure 9 shows that proportionally more $\mathrm{H} \alpha$ flux is emitted by the low-velocity component than that component in either $[\mathrm{N} \mathrm{II}]$ or $[\mathrm{S} \mathrm{II}]$; this is the pattern expected for a precursor.

Similar patterns are found in the $\mathrm{E}$ extension of the $\mathrm{S}$ shock complex. Between points 1 and 2 in Figure $7 a$ the [O III] $\lambda 5007$ emission line profiles are bright when the $\mathrm{H} \beta$ profiles are faint and vice versa.

\subsection{Properties of the Putative Mach Disk}

Inserts $(a)$ and $(b)$ in Figure 10 show that the $\mathrm{N}$ and $\mathrm{S}$ shocks both envelop a compact nebula that is detached from the main shock. Spectra of the $S$ feature show the highest velocity dispersion of the entire $S$ shock complex, making it an excellent candidate for the Mach disk. The corresponding feature in the $\mathrm{N}$ complex may also be a Mach disk, but we need spectra to confirm this. We therefore restrict our discussion to the detached knot in the $S$ complex. Interpretations other than a Mach disk are possible for this knot. For example, the large velocity disper- 
sion might instead be the signature of ablata stripped from a dense cloud swept up in the outflow.

In the steady state, working surface model, a Mach disk should have roughly the same radial velocity as the apex of the bow shock. This is observed in galactic Herbig-Haro objects (Morse et al. 1993) and is what we find in the $\mathrm{S}$ complex (Fig. 7). The large velocity dispersion may arise from nonsteady conditions or backwash of the thermalized jet material after it has flowed through the Mach disk.

Inserts in Figure 10 and Figure 5 show that the knot stands off from the bow shock by more than 2 WFC pixels $\left(\gtrsim 0^{\prime \prime} .2=7 \mathrm{pc}\right)$, has lateral extent 0.5 , and appears as a cone or paraboloid with apex toward the fainter half of the bow shock. We found $A_{V}=1.6$ on average over the knot. From this image, we attribute $\sim 60 \%$ of the summed count rate of the putative Mach disk to its $\mathrm{H} \alpha$ emission, $1.28 e^{-1}$ $\mathrm{s}^{-1}=8 \times 10^{-16} \mathrm{ergs} \mathrm{s}^{-1} \mathrm{~cm}^{-2}$, so the dereddened $L_{\mathrm{H} \alpha}=$ $1.5 \times 10^{37} \mathrm{ergs} \mathrm{s}^{-1}$. Taking the knot as a filled cone of height 0 ".40 and base radius 0.25 , we get $V=3.2 \times 10^{58}$ $\mathrm{cm}^{3}$. In case $\mathrm{B}$ recombination conditions we therefore obtain $n_{e}=40 f^{-1 / 2} \mathrm{~cm}^{-3}$, and $M_{\text {ion }} \approx 10^{3} \sqrt{f} M_{\odot}$.

Figure $7 b$ (left) shows obvious differences between the spectra of the knot and the S bow shock only in the [O III] $\lambda 5007$ line profiles. More emission is found at low velocities than at high velocities in [O III] than e.g., [N II]. Again, this is the expected behavior for gas associated with a Mach disk. For the geometry derived above, the highest velocity gas in the backflow should impinge near the apex of the bow shock, which has a correspondingly small emitting volume. The bow-shock models in Ferruit et al. (1997) show that lower excitation lines such as [N II] will have broader profiles than those with higher excitation such as [O III]. This is the pattern we observe. It will be interesting to see if this pattern persists in spectra with higher spatial resolution because our Herbig-Haro program (e.g., Morse et al. 1992) uncovered clear differences in $V_{s}$ hence ionization for those Mach disks. More complete spectra to establish the advance speed of the jet head $V_{\text {head }}$ will constrain the jet speed $V_{\text {jet }}$, because $V_{\text {head }}=\eta^{1 / 2} V_{\text {jet }} /\left(1+\eta^{1 / 2}\right)$, where $\eta$ is the density contrast between light jet fluid and denser ISM.

\subsection{Photoionization}

The spatio-kinematic structure of the bow-shock model agrees well with our spectra. However, the conclusion that the shocks are self-luminous rests on several uncertain assumptions, mainly poorly constrained gaseous filling factors $f$. We therefore now consider the possibility that the arcuate features, including the bow shock, are swept up by the outflow, but that they are photoionized.

\subsubsection{Hot Stars}

First, are hot stars viable sources of ionization? The WFPC2 continuum mosaic image (Fig. 4) resolves the brightest stars, but there is no sign of the O5-6 stars (average density one per $0.1 \mathrm{kpc}$ along the jet) postulated (Stüwe et al. 1992) to ionize the anomalous arms and (Shields 1992) to generate their LINER spectrum (CMV). Nor does the color map (Fig. 6b) show any substantial variation other than in the region found by Dutil et al. (1995), which CMV showed was produced by reduced reddening rather than a change in stellar population. However, Stüwe et al. note that molecular gas may obscure young stars, and indeed the continuum image does show considerable dust along and across the anomalous arms closer to the nucleus. Hence, establishing the ionizing influence of stars awaits high-resolution IR images that go deeper than those of Chary et al. (2000), or radio continuum measurements of thermal emission.

\subsubsection{Photoionization by the Galaxy Nucleus}

Does the AGN ionize extranuclear structures? Several ionized arcs other than the $\mathrm{N}$ and $\mathrm{S}$ complexes are evident in our HST image (Fig. 10) $\mathrm{N}$ of the nucleus, where Wilkes et al. (1995) and Barth et al. (1999) have detected polarized continuum emission. Some of these and the bright $\mathrm{W}$ half of the "Nuclear loop" discussed in $\S 3.6 .4$ end abruptly, as would be expected if their extremities outline the edges of an "ionization cone" that intersects the galaxy disk. The W half of the nuclear loop is also known (CMV) to have a large [O III] $\lambda 5007 / \mathrm{H} \alpha$ ratio $(1.25 \pm 0.3$ dereddened), characteristic of features that are embedded in ionization cones (Wilson \& Tsvetanov 1994). The cone is not prominent away from the nuclear loop, and the illuminated chord is quite narrow compared to other cones (Wilson \& Tsvetanov 1994), but such effects might result from the $\approx 70^{\circ}$ angle between the spin axis of the accretion disk (hence, plausibly, the axis of the ionization cone) and the plane of the galaxy disk.

\subsubsection{Probing the AGN Radiation Field}

We probed the AGN photoionizing field by using the fluxes of nebulae with small velocity deviations from galaxy rotation. An arc at radius $15^{\prime \prime}$ is labeled at the top of Figure 5; the FP velocity field of CWT (their Fig. 2c) shows it to have no significant deviation from nearby disk velocities. Dimensioned 3". $6=126$ pc long by 0".34 = 12 pc FWHM (resolved in the PC image) and assuming this delineates an unreddened box of height $\approx 10 \mathrm{pc}$ hence volume $4.1 \times 10^{59}$ $\mathrm{cm}^{3}$, we obtained $L_{\mathrm{H} \alpha}=3.4 \times 10^{37} \mathrm{ergs} \mathrm{s}^{-1}$ from the arc, so $n_{e}=1.6 f^{-1 / 2} \mathrm{~cm}^{-3}$. We estimate that the arc spans $4.3 \times 10^{-4}$ of the sky seen from the nucleus. The case B recombination rate in a photoionized arc is the arrival rate of ionizing photons from the nucleus, $N=7.3$ $\times 10^{11} L_{\mathrm{H} \alpha}=8 \times 10^{48}$ photon $\mathrm{s}^{-1}$ at $10^{4} \mathrm{~K}$. If the arc is ionized by a nuclear source that radiates isotropically, the number of ionizing photons emitted by the central engine is $Q_{\text {ion }}=1.9 \times 10^{52} / \varepsilon . \varepsilon$ is the photon fraction incident on the arc that is absorbed (local covering factor) and is relatively unconstrained.

The ionization parameter of the arc is $\Gamma=Q_{\text {ion }} /$ $4 \pi R^{2} c n=0.012 \sqrt{f} / \varepsilon$. This value, along with those for $M_{\text {ion }}$ and $Q_{\text {ion }}$ should be considered lower limits because we have not considered extinction between the arc and the nucleus, and the arc may be close to the (dusty) galaxy disk. The color map (Fig. $6 b$ ) shows little variation in this region. To constrain $\sqrt{f} / \varepsilon$ we would normally use the $[\mathrm{O}$ III $] /[\mathrm{O}$ II] flux ratio which is proportional to $\Gamma$ but insensitive to the ionizing spectrum for optically thick clouds (Penston et al. 1990). However, [O $\mathrm{II}] \lambda 3727$ in the arc is as yet unmeasured.

The spectral properties of the extranuclear arc are consistent with values derived by others for the nucleus. Based on optical emission line flux ratios, Stüwe et al. (1992) have argued for a central ionizing continuum of form $F_{v} \propto v^{-1.2}$ with $Q_{\text {ion }} \approx 10^{52} \mathrm{~s}^{-1}$. Wilkes et al. (1995) mapped polarized light $\mathrm{N}$ of the nucleus, and fit the polarized (presumed scattered nuclear) continuum adequately with the AGN form $F_{v} \propto v^{-1.1 \pm 0.2}$ in the range $415-800 \mathrm{~nm}$; see also Barth et 
al. (1999). At the hot spot nebulae we obtained $\Gamma \approx$ $0.14 \sqrt{f} /(n \varepsilon)$, which is 12 times the value we derived for the arc. The nebulae are at greater radii than the arc, so it is plausible that the incident spectrum is hardened by absorption compared to what the arc sees. DS obtain $\Gamma \simeq 0.01$ for a planar, semi-infinite shock with $V_{S}=500 \mathrm{~km} \mathrm{~s}^{-1}$, which is comparable to our inferred value if $\sqrt{f} / \varepsilon \approx 0.1$. Evidently, the AGN does photoionize extranuclear structures.

\subsubsection{AGN Ionization of the Hot Spot Nebulae}

We assume that the deprojected distances from the AGN to the shock complexes are $15 \%$ larger than observed to account for their estimated $30^{\circ}$ inclination to our viewpoint, $28^{\prime \prime}$ and $57^{\prime \prime}$, respectively, for $\mathrm{S}$ and $\mathrm{N}$ which both present diameter $5^{\prime \prime}$ to the nucleus. The fractions of solid angle that are subtended by the bow shocks at the nucleus are $2 \times 10^{-3}$ and $5 \times 10^{-4}$, respectively. They would therefore have $L_{H \alpha}=5.4 \times 10^{37}$ and $1.4 \times 10^{37}$ ergs s$^{-1}$ if photoionized by an isotropically emitting central engine. These values are $50 \%$ and $30 \%$ of the values obtained from our spectra. The flux discrepancy for the $\mathrm{N}$ shock exceeds the uncertainties of its reddening correction, and it is plausibly out of the disk. Therefore, the $\mathrm{N}$ nebula may be selfluminous. The $\mathrm{S}$ hot spot nebula could be photoionized by the AGN, depending on the dust distribution at the AGN and along the paths to the nebulae.

\subsection{Future Work on the Hot Postshock Gas}

As Allen et al. (1998) have emphasized, more near-UV resonance lines than optical lines are emitted from the hot, postshock gas than from the photoionized precursor. Unfortunately, the large reddening of both shock complexes precludes UV spectra. However, postshock gas is effectively traced with soft X-rays. Viewed with the ROSAT HRI, emission concentrates along the anomalous arms (CWD); ROSAT PSPC spectra of these regions are thermal with $T=0.3-0.5 \mathrm{keV}$ and have little photoelectric absorption beyond that of the Galactic screen (Pietsch et al. 1994; Vogler \& Pietsch 1998). Chandra X-ray Observatory (CXO) spectra will greatly improve spatial and spectral resolution of the arms in soft X-rays. A long integration is scheduled with the HRI grating spectrometer, and there is also GTO time with the ACIS spectrometer. Emission from hot, postshock gas should delineate a clear jet cocoon.

\section{THE GLOBAL GEOMETRY OF THE DISK-JET INTERACTION}

In this section we argue that the active jet is linked to the anomalous arms by precession of the central engine. We characterize the interaction with the galaxy gas disk.

\subsection{The Inner Jet}

According to van Albada (1980), H I kinematical data show that the large-scale disk of NGC 4258 is inclined at $i=72^{\circ}$ to the line of sight, and the P.A. of its major axis is at $148^{\circ}$. The VLBI images show that, at the scale of about 0.1 pc, the jet is aligned at P.A. $-3^{\circ}$, so the position angle $\delta$ between the apparent major axis of the galaxy and the radio jet projected on the plane of the sky is $\delta=151^{\circ}$. The $\mathrm{N}$ jet is the nearest to the line of sight and is inclined to the line of sight. by $\phi=83^{\circ}$. Hence, we conclude that the current jet direction relative to the axis of the galaxy, $\beta$, is given by $\cos \beta=\sin \delta \sin i \sin \phi+\cos i \cos \phi$. Substituting values gives $\beta=60^{\circ}$. If this jet had fixed orientation, then it could never have interacted with gas in the galaxy disk to produce the shock complexes and entrainment flows described in this paper. The inevitable conclusion is therefore that the jet is precessing in its direction and has recently moved a long way out of the plane of the galaxy.

\subsection{The Bow-Shock Region}

The $\mathrm{N}$ and $\mathrm{S}$ shock complexes represent the clearest (and presumably) most recent interactions between the jets and disk gas. The shocked gas is presumably being forced farther below the disk plane in the $\mathrm{S}$ (to give the observed redshift) and up and out of the disk plane in the $\mathrm{N}$ (where blueshifted gas is seen), Figure 14. The position angle of the interaction direction is $-5^{\circ} \pm 1^{\circ}$. Assuming that the interaction occurs within a scale height of the midplane, we can use the above equation to solve for the past jet inclination to the line of sight, $\beta=34^{\circ}$ ( $\mathrm{S}$ jet nearest). The jet must therefore have precessed by more than $60^{\circ}$ since it generated the bow shocks (Fig. 14). The observed angle between the present jet direction and the direction of the galaxy major axis $\theta_{\text {app }}=29^{\circ}$. Deprojecting this $\left(\tan \theta=\tan \theta_{\text {app }} / \cos i\right)$ gives a jet angle $\theta=61^{\circ}$ from the major axis in the plane of the galaxy.

Because the shock complexes have become radiative during the time that the jet has precessed to its current orientation, we can obtain a lower bound on the precession timescale. We assume that jet properties do not depend on time, and ignore differences in filling factor for the precursor gas as the jet propagates. The $\mathrm{S}$ bow shock was estimated in $\S 4.1 .5$ to have preshock density $\sim 2.6 \mathrm{~cm}^{-3}$ and shock velocity $\sim 300 \mathrm{~km} \mathrm{~s}^{-1}$. Such a shock will require $\sim 1.5 \times 10^{4} \mathrm{yr}$ to cool to recombine half of the gas, and $\sim 4 \times 10^{4}$ yr to recombine completely. During this time the jet has precessed through $\sim 60^{\circ}$ as traced by the deprojected extent of the kinematically disturbed emission in Figure 7 (including the "bubble" region). Thus, the precession timescale must be more than $2.5 \times 10^{5}$ yr. We note that the timescale in which the jet precesses by of order the warp in the accretion disk, $\approx 10^{\circ}$, is on the order of $3-10$ orbits at the outer and inner radii, respectively. The maser accretion disk may therefore extend to only the observed $0.29 \mathrm{pc}$ outer radius. Only a stable disk can form masers, and larger orbits would be too slow to be stable during a smooth precessional cycle.

Note that, although the jet has precessed through a fairly large angle since it last interacted with disk gas, its position angle on the sky has changed by only a few degrees. This implies that the precession axis lies fairly close to the sky plane in the E-W or SE-NW direction and that the precession angle is large (but inferred in other outflow systems, e.g., NGC 3516; Veilleux et al. 1993), approaching $90^{\circ}$.

\subsection{The Disk Interaction Region and Anomalous Arms}

Consider what will occur as a fast jet precesses into the disk of a galaxy. Initially it will interact with disk gas at large scale heights, driving a highly oblique shock front toward the plane, and entraining disk gas into jet material. When the mass-loaded jet has been slowed sufficiently, it acts somewhat like a flame thrower: buoyancy will take over, lofting a bubble of hot gas and entrained cooler gas out of the disk. Precession drives the jet ever deeper toward the midplane of the disk, decreasing the distance the jet propagates before buoyancy dominates. Eventually, the jet 


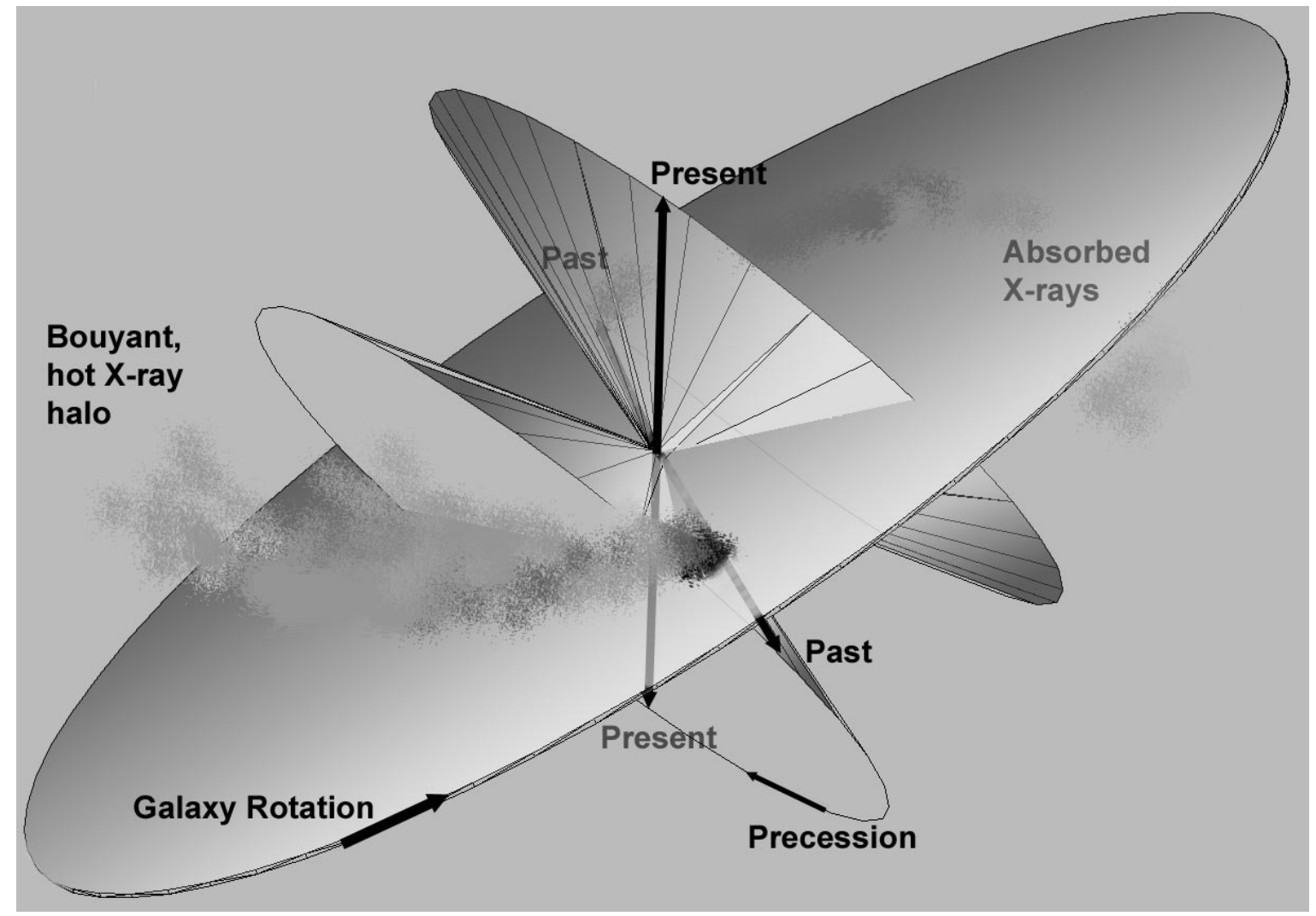

FIG. 14.-Possible orientations of the jets in NGC 4258 as presented to us at different times in the past. Precession cones are approximate because they are loosely constrained (see text). The $\mathrm{S}$ jet is currently well below the disk, and the $\mathrm{N}$ jet above. The sense of precession has driven, e.g., the right-hand jet up and out of the disk. In our model, both jets intersected the disk when the bow shocks were formed. The galaxy disk is shown inclined at $72^{\circ}$. The $\mathrm{E}$ anomalous arm is shown above the galaxy disk, and the $\mathrm{W}$ arm below.

breaks out on the far side of the galaxy. This may happen quite suddenly when the jet is already angled appreciably below the midplane.

This interaction is the most likely explanation for the anomalous arms in NGC 4258, which may be interpreted as recombining gas along the surface of two expanding bubbles of hot low-density gas excavated by the jets. In this picture, the passage of the interaction region of the $S$ jet from the near top side of the galaxy into the disk plane is traced by the decreasing blueshift relative to the galaxy disk from 600 to $100 \mathrm{~km} \mathrm{~s}^{-1}$ and increasing reddening from $A_{V}=1$ to $\sim 1.7$ of the luminous shocked gas seen from $\mathrm{E}$ to W along the spectral slit in Figure 7 from region 3 to 1 . The strongly redshifted and reddened bow-shock structure can be interpreted as arising from gas back-flowing from the shock breakthrough on the far bottom side of the galaxy.

Somewhat the same structure may be evident near the $\mathrm{N}$ bow shock, except here gas is slightly redshifted as it stalls in the disk, and the bow-shock feature is instead blueshifted as it flows out of the plane on the far side of the galaxy. These orientations of the disk blowout are consistent with the patterns of X-ray absorption mapped by Vogler \& Pietsch (1998). In the $S$, the anomalous arm is oriented initially at $\sim$ P.A. $139^{\circ}$, and in the N, along P.A. $312^{\circ}$. These angles are close to the P.A. of the galaxy major axis, so the arms must be propagating nearly in the plane of the sky, see Figure 14. Such orientation explains why the anomalous arms generally show only modest oscillations of their velocity centroids $\left( \pm 50-75 \mathrm{~km} \mathrm{~s}^{-1}\right)$ around the galaxy rotation.
It is significant that the region of strongest radio emission in the $\mathrm{N}$ anomalous arm coincides with a dusty complex seen in projection (Fig. 4, top insert) and where the $\mathrm{N}$ anomalous arm bifurcates. Here a dense cloud is clearly obstructing, deflecting, and dividing the entrainment flow. At larger galactocentric radii the southern of these arms again bifurcates. In the TTF images its line emission becomes more prominent (Fig. $2 a$ and $2 b$ ) as the surface brightness of the diffuse $\mathrm{H} \alpha$ emission in the galaxy disk drops with increasing galactocentric radius. The short recombination time $(\S 3.1)$ compared to the dynamical time of the anomalous arms suggests a continued interaction with disk gas.

\subsection{What Role for Bar Shocks?}

Cox \& Downes (1996) found that most of the CO flux in their IRAM $30 \mathrm{~m}$ telescope map concentrates along the inner anomalous arms, at the P.A. of what we have shown in this paper to be the active jet. They argued that the large $\mathrm{H}_{2}$ mass $\left(10^{9} \quad M_{\odot}\right.$ assuming the standard $\mathrm{CO} / \mathrm{H}_{2}$ conversion) makes it implausible that the anomalous arms were ejected from the nucleus in an explosion (van der Kruit et al. 1972) or as jets; this mass, its distribution along the bar, and its pattern of velocity deviations from pure rotation, are consistent with those found in other barred galaxies. For example, Figure 8 shows a discontinuity of 100 $\mathrm{km} \mathrm{s}^{-1}$ as our spectral slit crosses the NW anomalous arm, a value and pattern consistent with a bar shock. Cox \& Downes attributed most of the kinematic activity, and indeed the X-ray and radio emitting anomalous arms, to 
bar shocks that stream gas close to what we have identified as the axis of the active jet.

A different interpretation follows from the more sensitive CO images of Neininger (1999) made with the IRAM interferometer. These spectra resolve the single bar seen by Cox $\&$ Downes into emission ridges that straddle the active flow within $30^{\prime \prime}$ radius; this result extends to larger radii the earlier detection of a channel reported by Plante et al. (1991). This image suggests that the CO distribution is intimately connected to the active jet flow.

CWT showed that an oval distortion is needed to explain the $\mathrm{H} \alpha$ velocity field of the galaxy disk. This has a different major axis orientation $\left(15^{\circ} \pm 2^{\circ}\right)$ than the bar proposed by Cox \& Downes. The velocities of its shocks, discussed in $\S 3.6 .5$, can explain the abrupt changes in direction of various prominent dust lanes (Fig. 4) but are too low to generate the X-ray emission of the anomalous arms. There are few kinematic manifestations of bar shocks at other P.A.'s (CWT), not what one would expect if the bar flow is as influential and oriented as Cox \& Downes propose. Our observation that all high-velocity features within $50^{\prime \prime}$ radius are in a precession pattern that culminates at the P.A. of the active flow is not explained by the Cox \& Downes hypothesis.

\section{CONCLUSIONS}

The radio data in this paper provide the first evidence for a tightly collimated outflow to $1-2 \mathrm{kpc}$ radii in NGC 4258 from the established accretion disk that is bound to the central engine. Two well-defined radio hot spots are projected along the flow axis. HST images show that these structures are enveloped by sharp edged, emission line nebulae. Our WHT spectra have confirmed that these nebulae are shock fronts. The spatial distribution of kinematics and extinction of the $\mathrm{S}$ shock favor a location near the galaxy disk and gas backflow from a working surface. While the outflow projects on the sky to within $\approx 10^{\circ}$ of the spin axis of the nuclear accretion disk, the kinematics and energetics at the $\mathrm{S}$ bow shock imply motion near the disk plane. The large angular difference implies that the active jet has recently (within a recombination time) precessed by a large angle out of the galaxy disk plane, and that the anomalous arms trace previous jet orientations. Excitation ratios at the bow apex, the detailed kinematics (when interpreted as a backflow), and the overall $\mathrm{H} \alpha$ FWZI across the full structure are all consistent with shock velocities $V_{s} \approx 350$ $\pm 100 \mathrm{~km} \mathrm{~s}^{-1}$.

Unique among the handful of bow shocks resolved in AGN NLRs, those in NGC 4258 are sufficiently energetic that their entire ionization may arise locally. Both complexes are large enough on the sky for detailed study with the spectrometers on $C X O$. These shocks and their putative Mach disks are thus important laboratories for study of high-velocity shocks and jet entrainment, topics with broad astrophysical application.

We thank J. Bland-Hawthorn for obtaining the TTF images, and the referee for detailed comments that improved the organization of this paper. G. C., C. D., L. G., A. A., and J. M. are supported in this work by NASA through grant number GO-6563 from the Space Telescope Science Institute, which is operated by AURA, Inc., under NASA contract NAS 5-26555.
Allen, M., et al. 1998, ApJ, 493, 571

Barth, A., J., et al. 1999, AJ, 118, 1609

Bicknell, G. V., Dopita, M. A., Tsvetanov, Z. I., \& Sutherland, R. S. 1998, ApJ, 495, 680

Biretta, J. A. 1996, WFPC2 Instrument Handbook (Baltimore: STScI)

Bland-Hawthorn, J. 1995, in IAU Colloq. 149, Tridimensional Optical Spectroscopic Methods in Astrophysics, ed. G. Comte \& M. Marcelin (San Francisco: ASP), 72

Bland-Hawthorn, J., \& Jones, D. H. 1998, Proc. SPIE, 3555, 855

Cecil, G. 1988, ApJ, 329, 38

Cecil, G., Morse, J. A., \& Veilleux, S. 1995a, ApJ, 452, 613 (CMV)

Cecil, G., Wilson, A. S., \& De Pree, C. 1995b, ApJ, 440, 181 (CWD)

Cecil, G., Wilson, A. S., \& Tully, R. B. 1992, ApJ, 390, 365 (CWT)

Chary, R., Becklin, E. E., Evans, A. S., Naugebauer, G., Scoville, N. Z., Matthews, K., \& Ressler, M. E. 2000, ApJ, 531, 756

Cox, P., \& Downes, D. 1996, ApJ, 473, 219

Dopita, M. A., \& Sutherland, R. S. 1995, ApJ, 455, 468 (DS)

Dutil, Y., Beauchamp, D., \& Roy, J.-R. 1995, ApJ, 444, 85

Ferruit, P., Binette, L., Sutherland, R. S., \& Pecontal, E. 1997, A\&A, 322, 73

Ferruit, P., Wilson, A. S., Falcke, H., Simpson, C., Pécontal, E., \& Durret, F. 1999, MNRAS, 309, 1

Hartigan, P., Raymond, J., \& Hartmann, L. 1987, ApJ, 316, 323 (HRH)

Hartigan, P., Raymond, J., \& Meaburn, J. 1990, ApJ, 362, 624 (HRM)

Herrnstein, J. R., et al. 1996, ApJ, 468, L17

Herrnstein, J. R., et al. 1997, ApJ, 475, L17

Herrnstein, J. R., et al. 1999, Nature, 400, 539

Hummel, E., Krause, M., \& Lesch, H. 1989, A\&A, 211, 266

Leahy, J. P. 1991, in Beams and Jets in Astrophysics, ed. P. A. Hughes (Cambridge: Cambridge Univ. Press), 100

\section{REFERENCES}

Miyoshi, M., et al. 1995, Nature, 373, 127

Morse, J. A., et al. 1992, ApJ, 399, 231 1993, ApJ, 410, 764

Neininger, N. 1999, preprint (astro-ph/9903230)

Penston, M. V., et al. 1990, A\&A, 236, 53

Pietsch, W., Vogler, A., Kahabka, P., Jain, A., \& Klein, U. 1994, A\&A, 284, 386

Plante, R. L., Lo, K.-Y., Roy, J. R., Martin, P., \& Noreau, L. 1991, ApJ, 381,110

Raga, A., \& Bohm, K. H. 1986, ApJ, 308, 829

Rubin, V. C., \& Graham, J. A. 1990, ApJ, 362, L5

Shields, J. 1992, ApJ, 399, L27

Steffen, W., et al. 1997, ApJ, 491, L73

Stüwe, J. A., et al. 1992, A\&A, 261, 382

Taylor, D., Dyson, J. E., \& Axon, D. 1992, MNRAS, 255, 351

van Albada, G. D. 1980, A\&A, 90, 123

van der Kruit et al. 1972, A\&A, 21, 169

Veilleux, S., \& Bland-Hawthorn, J. 1996, ApJ, 479, 105

Whittle, M. 1985, MNRAS, 213, 189

Vogler, A., \& Pietsch, W. 1998, in IAU Colloq. 166, The Local Bubble and Beyond, ed. M. J. Freyberg \& J. Truemper (Berlin: Springer), 547

Williams, A. G. 1991, in Beams and Jets in Astrophysics, ed. P. A. Hughes (Cambridge: Cambridge Univ. Press), 342

Wilkes, B., et al. 1995, ApJ, 455, L16

Wilson, A. S., \& Raymond, J. C. 1999, ApJ, 513, L115

Wilson, A. S., \& Tsvetanov, Z. 1994, AJ, 107, 1227

Wilson, A. S., \& Ulvestad, J. 1987, ApJ, 319, 105 